\title{
Cyclic homology for bornological coarse spaces
}

\section{Luigi Caputi ${ }^{1}$}

Received: 22 November 2019 / Accepted: 10 July 2020 / Published online: 24 July 2020 (c) The Author(s) 2020

\begin{abstract}
The goal of the paper is to define Hochschild and cyclic homology for bornological coarse spaces, i.e., lax symmetric monoidal functors $\mathcal{X} \mathrm{HH}^{G}$ and $\mathcal{X} \mathrm{HC}^{G}$ from the category $G$ BornCoarse of equivariant bornological coarse spaces to the cocomplete stable $\infty$-category $\mathbf{C h}_{\infty}$ of chain complexes reminiscent of the classical Hochschild and cyclic homology. We investigate relations to coarse algebraic $K$-theory $\mathcal{X} K^{G}$ and to coarse ordinary homology $\mathcal{X} \mathrm{H}^{G}$ by constructing a trace-like natural transformation $\mathcal{X} K^{G} \rightarrow \mathcal{X} \mathrm{H}^{G}$ that factors through coarse Hochschild (and cyclic) homology. We further compare the forget-control map for $\mathcal{X} \mathrm{HH}^{G}$ with the associated generalized assembly map.
\end{abstract}

Keywords K-theory and homology · Algebraic Topology · Coarse Geometry

\section{Contents}

Introduction . . . . . . . . . . . . . . . . . . . . . . . . . . . 464

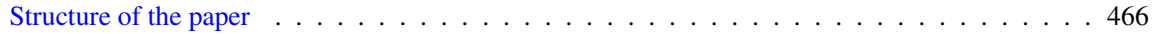

Conventions . . . . . . . . . . . . . . . . . . . . . . . 467

1 Equivariant coarse homotopy theory . . . . . . . . . . . . . . . . . . . . . . . . 467

1.1 Equivariant bornological coarse spaces . . . . . . . . . . . . . . . . . . . . 467

1.2 Equivariant coarse homology theories . . . . . . . . . . . . . . . . . 468

1.3 Coarse ordinary homology . . . . . . . . . . . . . . . . . . . . . . . 469

1.4 The category $V_{\mathbf{A}}^{G}(X)$ of controlled objects $\ldots \ldots \ldots \ldots \ldots \ldots \ldots \ldots$

2 Keller's cyclic homology for dg-categories . . . . . . . . . . . . . . . . . . . . . . 474

2.1 Dg-categories . . . . . . . . . . . . . . . . . . . . . . . . . 474

2.2 The $\infty$-category of mixed complexes . . . . . . . . . . . . . . . . . . . 475

2.3 Keller's cyclic homology . . . . . . . . . . . . . . . . . . . . . . . . . . . 477

3 Equivariant coarse Hochschild and cyclic homology . . . . . . . . . . . . . . . . . . . . 479

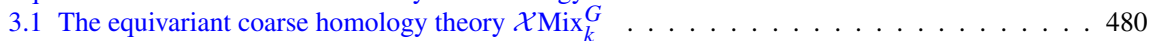

3.2 Coarse Hochschild and cyclic homology . . . . . . . . . . . . . . . . . . . 483

3.3 Comparison results and assembly maps . . . . . . . . . . . . . . . . . . . . 484

Communicated by Chuck Weibel.

$凶$ Luigi Caputi

luigi.caputi@ur.de

1 Fakultät für Mathematik, Universität Regensburg, Universitätsstraße 31, 93040 Regensburg, Germany 
4 From coarse algebraic $K$-theory to coarse ordinary homology . . . . . . . . . . . . . . . . . 486

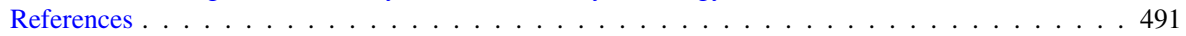

\section{Introduction}

Coarse geometry is the study of metric spaces from a large-scale point of view [27-30]. A new axiomatic and homotopic approach to coarse geometry and coarse homotopy theory has been recently developed by Bunke and Engel [3]. In this set-up, the main objects are called bornological coarse spaces [3, Definition 2.5], and every metric space is a bornological coarse space in a canonical way. In the equivariant setting, if $G$ is a group, $G$-bornological coarse spaces are bornological coarse spaces with a $G$-action by automorphisms [4, Definition 2.1]. Among various invariants of $G$ bornological coarse spaces we are interested in equivariant coarse homology theories, i.e., functors

\section{$E: G$ BornCoarse $\rightarrow \mathbf{C}$}

from the category $G$ BornCoarse of $G$-bornological coarse spaces to a cocomplete stable $\infty$-category $\mathbf{C}$, satisfying some additional axioms: coarse invariance, flasqueness, coarse excision and u-continuity [4, Definition 3.10]. Examples of coarse homology theories arise as coarsifications of locally finite homology theories [3]. Among other theories, there are coarse versions of ordinary homology and of topological $K$-theory [3], of equivariant algebraic $K$-homology and of topological Hochschild homology [2,4], and of Waldhausen's $A$-theory [6].

Classically, Hochschild and cyclic homologies have been defined as homology invariants of algebras [22], then extended to invariants of dg-algebras, schemes, additive categories and exact categories $[21,26]$. The aim of the paper is twofold: we construct coarse homology theories defining Hochschild and cyclic homology for bornological coarse spaces and then we study their relations to coarse algebraic $K$ theory and coarse ordinary homology. We remark that these coarse homology theories can be abstractly defined by using a universal equivariant coarse homology theory constructed by Bunke and Cisinski [2]. However, we choose to provide a more concrete construction with the hope that it might be more suitable for computations (see, e.g., the application to the construction of the natural transformation to coarse ordinary homology, Theorem 4.8). We now describe the main results of the paper.

Let $k$ be field and let $G$ be a group. We denote by $C_{*}^{\mathrm{HH}}$ and $C_{*}^{\mathrm{HC}}$ the chain complexes computing Hochschild homology and cyclic homology (of $k$-algebras) respectively. The $G$-bornological coarse space $G_{\mathrm{can} \text {, min }}$ denotes a canonical bornological coarse space associated to the group $G$ (see Example 1.2). Let $\mathbf{C h}_{\infty}$ be the $\infty$-category of chain complexes. The following is a combination of Theorem 3.11, Propositions 3.12, 3.14, Proposition 3.15:

Theorem A There are lax symmetric monoidal functors

$$
\mathcal{X} \mathrm{HH}_{k}^{G}: G \text { BornCoarse } \rightarrow \mathbf{C h}_{\infty} \text { and } \mathcal{X} \mathrm{HC}_{k}^{G}: G \text { BornCoarse } \rightarrow \mathbf{C h}_{\infty}
$$

satisfying the following properties: 
(i) $\mathcal{X} \mathrm{HH}_{k}^{G}$ and $\mathcal{X} \mathrm{HC}_{k}^{G}$ are $G$-equivariant coarse homology theories;

(ii) there are equivalences of chain complexes

$$
\mathcal{X} \mathrm{HH}_{k}^{G}(*) \simeq C_{*}^{\mathrm{HH}}(k) \text { and } \quad \mathcal{X} \mathrm{HC}_{k}^{G}(*) \simeq C_{*}^{\mathrm{HC}}(k)
$$

between the evaluations of $\mathcal{X} \mathrm{HH}_{k}^{G}$ and $\mathcal{X} \mathrm{HC}_{k}^{G}$ at the one-point bornological coarse space $\{*\}$, endowed with the trivial $G$-action, and the chain complexes computing Hochschild and cyclic homology of $k$;

(iii) there are equivalences

$$
\mathcal{X} \mathrm{HH}_{k}^{G}\left(G_{\text {can }, \min }\right) \simeq C_{*}^{\mathrm{HH}}(k[G] ; k) \quad \text { and } \quad \mathcal{X} \mathrm{HC}_{k}^{G}\left(G_{\text {can }, \min }\right) \simeq C_{*}^{\mathrm{HC}}(k[G] ; k)
$$

of chain complexes between the evaluations at the G-bornological coarse space $G_{\mathrm{can}, \min }$ and the chain complexes computing Hochschild and cyclic homology of the $k$-algebra $k[G]$.

The construction of the functors $\mathcal{X} \mathrm{HH}_{k}^{G}$ and $\mathcal{X} \mathrm{HC}_{k}^{G}$ uses a cyclic homology theory for dg-categories that satisfies certain additive and localizing properties in the sense of Tabuada [35]. This is Keller's cone construction

$$
\text { Mix : dgcat } \rightarrow \text { Mix }
$$

for dg-categories [21], defined as a functor from the category dgcat of small $\mathrm{dg}$ categories to Kassel's category Mix of mixed complexes [19]. Hochschild and cyclic homologies for dg-categories are then defined in terms of mixed complexes, consistently with the classical definitions for $k$-algebras [19]. We also consider the functor (with values in the category of small $k$-linear categories Cat $_{k}$ )

$$
V_{k}^{G}: G \text { BornCoarse } \rightarrow \text { Cat }_{k},
$$

that associates to every $G$-bornological coarse space $X$ a suitable $k$-linear category $V_{k}^{G}(X)$ of $G$-equivariant $X$-controlled (finite-dimensional) $k$-vector spaces [4, Definition 8.3]; a $k$-linear category is a dg-category in a standard way. We prove that the following functor

$$
\mathcal{X} \operatorname{Mix}_{k}^{G}: \text { B BornCoarse } \stackrel{V_{k}^{G}}{\longrightarrow} \text { Cat }_{k} \stackrel{\iota}{\longrightarrow} \text { dgcat }_{k} \stackrel{\text { Mix }_{\longrightarrow}}{\longrightarrow} \text { Mix } \stackrel{\text { loc }}{\longrightarrow} \text { Mix }_{\infty}
$$

(see Definition 3.1) with values in the cocomplete stable $\infty$-category of mixed complexes Mix $\mathbf{x}_{\infty}$ is a coarse homology theory (Theorem 3.2). Coarse Hochschild $\mathcal{X} \mathrm{HH}_{k}^{G}$ and coarse cyclic homology $\mathcal{X} \mathrm{HC}_{k}^{G}$ are then defined by post-composition of the Hochschild and cyclic homology functors for mixed complexes with the functor $\mathcal{X} \mathrm{Mix}_{k}^{G}$ (see Definition 3.10).

Let $\mathbf{S p}$ be the $\infty$-category of spectra. The main reason of defining coarse versions of Hochschild and cyclic homology is to relate them to (the $\mathbf{S p}$-valued) equivariant coarse 
algebraic $K$-homology $\mathcal{X} K_{k}^{G}: G$ BornCoarse $\rightarrow$ Sp [4, Definition 8.8], because classically algebraic $K$-theory comes equipped with trace maps (e.g., the Dennis trace map from algebraic $K$-theory of rings to Hochschild homology, or the refined version, the cyclotomic trace, from the algebraic $K$-theory spectrum to the topological cyclic homology spectrum) to cyclic homology theories, and these trace maps have been of fundamental importance in its understanding [5,13]. Inspired by the classical case, we define trace maps to equivariant coarse Hochschild and cyclic homology and from equivariant coarse Hochschild and cyclic homology to equivariant coarse ordinary homology $\mathcal{X} \mathrm{H}^{G}:$ GBornCoarse $\rightarrow$ Sp (see Propositions 4.9, 4.10, Theorem 4.8):

Theorem B (1) The classical Dennis trace map induces a natural transformation of equivariant coarse homology theories:

$$
\mathrm{K} \mathcal{X}_{k}^{G} \rightarrow \mathcal{X} \mathrm{HH}_{k}^{G}
$$

(2) There is a natural transformation

$$
\Phi_{\mathcal{X H H}}^{G}: \mathcal{X} \mathrm{HH}_{k}^{G} \rightarrow \mathcal{X} \mathrm{H}^{G}
$$

of G-equivariant coarse homology theories, which induces an equivalence of spectra when evaluated at the one-point space $\{*\}$.

By composition, we get a natural transformation,

$$
K \mathcal{X}_{k}^{G} \rightarrow \mathcal{X H H}_{k}^{G} \rightarrow \mathcal{X} \mathrm{H}^{G}
$$

that factors through coarse Hochschild homology. The advantage of this transformation is that equivariant coarse ordinary homology $\mathcal{X} \mathrm{H}^{G}$ is defined in terms of equivariant locally finite controlled maps $X^{n+1} \rightarrow k$ (see Definition 1.6) and it might be suitable for computations of coarse $K$-theory classes.

We conclude with some applications to assembly maps. One of the main applications of coarse homotopy theory is within the studying of assembly map conjectures. We then provide a comparison result between the forget-control maps for equivariant coarse Hochschild and cyclic homology and the associated assembly maps (see Proposition 3.18).

\section{Structure of the paper}

In Sect. 1 we review the basic definitions in coarse homotopy theory: bornological coarse spaces, coarse homology theories and categories of controlled objects. In Sect. 2, we introduce the (cocomplete stable $\infty$-category) of mixed complexes and Keller's definition of cyclic homology. In Sect. 3 we define the functors $\mathcal{X} \operatorname{Mix}_{k}^{G}$, $\mathcal{X} \mathrm{HH}_{k}^{G}$ and $\mathcal{X} \mathrm{HC}_{k}^{G}$ and we prove that they are equivariant coarse homology theories. In the last Sect. 4, we construct the natural transformations from coarse algebraic $K$-homology to coarse ordinary homology factoring through coarse Hochschild homology. 


\section{Conventions}

We freely employ the language of $\infty$-categories. More precisely, we model $\infty$ categories as quasi-categories [9,24,25]. When not otherwise specified, $G$ will denote a group, $k$ a field, $\otimes$ the tensor product over $k$. Without further comments, we always consider an additive category as a dg-category in the canonical way.

\section{Equivariant coarse homotopy theory}

The main purpose of this section is to recollect the basic definitions in coarse homotopy theory and the notations needed in Sects. 3 and 4. We describe the category $G$ BornCoarse of $G$-equivariant bornological coarse spaces and the associated $G$ equivariant coarse homology theories, we give the examples of coarse ordinary homology and coarse algebraic $K$-homology, together with the properties of the category of controlled objects $V_{k}^{G}(X)$. We refer to [3, Sect. 2] and [4, Sects. 2 and 3] for a comprehensive introduction to (equivariant) coarse homotopy theory.

\subsection{Equivariant bornological coarse spaces}

A bornology on a set $X$ is a subset $\mathcal{B} \subseteq \mathcal{P}(X)$ of the power set of $X$ that is closed under taking subsets and finite unions, and such that $X=\cup_{B \in \mathcal{B}} B$. Its elements are called bounded sets.

A coarse structure on a set $X$ is a subset $\mathcal{C} \subseteq \mathcal{P}(X \times X)$ which contains the diagonal $\Delta_{X}:=\{(x, x) \in X \times X \mid x \in X\}$ and is closed under taking subsets, finite unions, inverses, and compositions. The elements of $\mathcal{C}$ are called entourages. If $\mathcal{U}$ is an entourage of a coarse space $X$ and $B$ is any subset of $X$, the $\mathcal{U}$-thickening of $B$ is the subset of $X$ :

$$
\mathcal{U}[B]:=\{x \in X \mid \exists b \in B,(x, b) \in \mathcal{U}\} \subseteq X
$$

A bornology $\mathcal{B}$ and a coarse structure $\mathcal{C}$ on a set $X$ are compatible if for every $U \in \mathcal{C}$ and every $B \in \mathcal{B}$ the controlled thickening $U[B]$ belongs to the family $\mathcal{B}$.

Definition 1.1 [3, Definition 2.5] A bornological coarse space is a triple $(X, \mathcal{C}, \mathcal{B})$ given by a set $X$, a bornology $\mathcal{B}$ and a coarse structure $\mathcal{C}$ on $X$, such that $\mathcal{B}$ and $\mathcal{C}$ are compatible.

Morphisms of bornological coarse spaces are maps such that pre-images of bounded sets are bounded sets and images of entourages are entourages. A $G$-bornological coarse space [4, Definition 2.1] is a bornological coarse space $(X, \mathcal{C}, \mathcal{B})$ equipped with a $G$-action by automorphisms such that the set of $G$-invariant entourages $\mathcal{C}^{G}$ is cofinal in $\mathcal{C}$. We denote by $G$ BornCoarse the category of $G$-bornological coarse spaces and $G$-equivariant, proper controlled maps. When clear from the context, we shortly write $X$ for denoting a $G$-bornological coarse space $(X, \mathcal{C}, \mathcal{B})$. 
Example 1.2 (i) Let $G$ be a group, $\mathcal{B}_{\min }$ be the minimal bornology on its underlying set and let $\mathcal{C}_{\text {can }}:=\left\langle\left\{G(B \times B) \mid B \in \mathcal{B}_{\min }\right\}\right\rangle$ be the coarse structure on $G$ generated by the $G$-orbits. The space $G_{\text {can,min }}:=\left(G, \mathcal{C}_{\text {can }}, \mathcal{B}_{\text {min }}\right)$ is a $G$-bornological coarse space.

(ii) Let $X$ be a $G$-bornological coarse space and let $Z$ be a $G$-invariant subset of $X$. Then, the triple $Z_{X}:=\left(Z, \mathcal{C}_{Z}, \mathcal{B}_{Z}\right)$ is a $G$-bornological coarse space, where $\mathcal{C}_{Z}:=\{(Z \times Z) \cap U \mid U \in \mathcal{C}\}$ and $\mathcal{B}_{Z}:=\{Z \cap B \mid B \in \mathcal{B}\}$.

(iii) Let $U$ be a $G$-invariant entourage of $X$. If $\mathcal{C}_{U}$ denotes the coarse structure on $X$ generated by $U$, then $X_{U}:=\left(X, \mathcal{C}_{U}, \mathcal{B}\right)$ is a $G$-bornological coarse space. Observe that there is a canonical morphism $X_{U} \rightarrow X$.

\subsection{Equivariant coarse homology theories}

Let $f_{0}, f_{1}: X \rightarrow X^{\prime}$ be morphisms between bornological coarse spaces. We say that $f_{0}$ and $f_{1}$ are close to each other if the image of the diagonal $\left(f_{0}, f_{1}\right)\left(\Delta_{X}\right)$ is an entourage of $X^{\prime}$. A morphism $f: X \rightarrow X^{\prime}$ is an equivalence if there exists an inverse $g: X^{\prime} \rightarrow X$ such that the compositions $g \circ f$ and $f \circ g$ are close to the respective identity maps. Two morphisms between $G$-bornological coarse spaces are close to each other if they are close as morphisms between the underlying bornological coarse spaces.

Definition 1.3 [4, Definition 3.8] A $G$-bornological coarse space $(X, \mathcal{C}, \mathcal{B})$ is called flasque if it admits a morphism $f: X \rightarrow X$ such that:

(i) $f$ is close to the identity map;

(ii) for every entourage $U$, the subset $\bigcup_{k \in \mathbb{N}}\left(f^{k} \times f^{k}\right)(U)$ is an entourage of $X$;

(iii) for every bounded set $B$ in $X$ there exists $k$ such that $f^{k}(X) \cap G B=\emptyset$.

Definition 1.4 [4, Definition 3.5 and 3.7] Let $(X, \mathcal{C}, \mathcal{B})$ be a $G$-bornological coarse space.

(1) A big family $\mathcal{Y}=\left(Y_{i}\right)_{i \in I}$ on $X$ is a filtered family of subsets of $X$ satisfying the following:

$$
\forall i \in I, \quad \forall U \in \mathcal{C}, \exists j \in I \text { such that } U\left[Y_{i}\right] \subseteq Y_{j}
$$

An equivariant big family is a big family consisting of $G$-invariant subsets.

(2) A pair $(Z, \mathcal{Y})$ consisting of a subset $Z$ of $X$ and of a big family $\mathcal{Y}$ on $X$ is called a complementary pair if there exists an index $i \in I$ for which $Z \cup Y_{i}=X$. It is an equivariant complementary pair if $Z$ is a $G$-invariant subset and $\mathcal{Y}$ is an equivariant big family.

Let $Z$ be a subset of $X$. If $\mathcal{Y}$ is a big family on $X$, then the intersection $Z \cap \mathcal{Y}:=$ $\left(Z \cap Y_{i}\right)_{i \in I}$ is a big family on $Z$. If $E: G$ BornCoarse $\rightarrow \mathbf{C}$ is a functor with values in a cocomplete $\infty$-category $\mathbf{C}$, we define the value of $E$ at the family $\mathcal{Y}$ as the filtered colimit $E(\mathcal{Y}):=\operatorname{colim}_{i \in I} E\left(Y_{i}\right)$. There is an induced map from $E(\mathcal{Y})$ to $E(X)$. Let $X_{U}$ be the $G$-bornological coarse space constructed in Example 1.2. 
Definition 1.5 [4, Definition 3.10] Let $G$ be a group and let $G$ BornCoarse be the category of $G$-bornological coarse spaces. Let $\mathbf{C}$ be a cocomplete stable $\infty$-category. A $G$-equivariant $\mathbf{C}$-valued coarse homology theory is a functor

\section{$E: G$ BornCoarse $\longrightarrow \mathbf{C}$}

with the following properties:

i. Coarse invariance: $E$ sends equivalences $X \rightarrow X^{\prime}$ of $G$-bornological coarse spaces to equivalences $E(X) \rightarrow E\left(X^{\prime}\right)$ of $\mathbf{C}$;

ii. Flasqueness: if $X$ is a flasque $G$-bornological coarse space, then $E(X) \simeq 0$;

iii. Coarse excision: $E(\emptyset) \simeq 0$, and for every equivariant complementary pair $(Z, \mathcal{Y})$ on $X$, the diagram

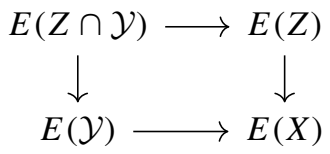

is a push-out square;

iv. u-continuity: for every $G$-bornological coarse space $(X, \mathcal{C}, \mathcal{B})$, the canonical morphisms $X_{U} \rightarrow X$ induce an equivalence $E(X) \simeq \operatorname{colim}_{U \in \mathcal{C}^{G}} E\left(X_{U}\right)$.

Examples of (equivariant) coarse homology theories are coarse ordinary homology (1.4) and coarse topological K-theory [3], coarse algebraic K-theory (Definition 1.19) and coarse topological Hochschild homology [2,4], coarse Hochschild and cyclic homology.

\subsection{Coarse ordinary homology}

Let $X$ be a $G$-bornological coarse space, $n \in \mathbb{N}$ a natural number, $B$ a bounded set of $X$, and $x=\left(x_{0}, \ldots, x_{n}\right)$ a point of $X^{n+1}$. We say that $x$ meets $B$ if there exists $i \in\{0, \ldots, n\}$ such that $x_{i}$ belongs to $B$. If $U$ is an entourage of $X$, we say that $x$ is $U$-controlled if, for each $i$ and $j$, the pair $\left(x_{i}, x_{j}\right)$ belongs to $U$.

An $n$-chain $c$ on $X$ is a function $c: X^{n+1} \rightarrow \mathbb{Z}$; its support $\operatorname{supp}(c)$ is defined as the set of points for which the function $c$ is non-zero:

$$
\operatorname{supp}(c)=\left\{x \in X^{n+1} \mid c(x) \neq 0\right\} .
$$

We say that an $n$-chain $c$ is $U$-controlled if every point $x$ of $\operatorname{supp}(c)$ is $U$-controlled. The chain $c$ is locally finite if, for every bounded set $B$, the set of points in $\operatorname{supp}(c)$ which meet $B$ is finite. An $n$-chain $c: X^{n+1} \rightarrow \mathbb{Z}$ is controlled if it is locally finite and $U$-controlled for some entourage $U$ of $X$.

Definition 1.6 Let $X$ be a bornological coarse space. Then, for $n \in \mathbb{N}, \mathcal{X} C_{n}(X)$ denotes the free abelian group generated by the locally finite controlled $n$-chains on $X$. 
We will also represent $n$-chains as formal sums $\sum_{x \in X^{n+1}} c(x) x$ that are locally finite and $U$-controlled. The boundary map $\partial: \mathcal{X} C_{n}(X) \rightarrow \mathcal{X} C_{n-1}(X)$ is defined as the alternating sum $\partial:=\sum_{i}(-1)^{i} \partial_{i}$ of the face maps $\partial_{i}\left(x_{0}, \ldots, x_{n}\right):=$ $\left(x_{0}, \ldots, \hat{x}_{i}, \ldots, x_{n}\right)$. The graded abelian group $\mathcal{X} C_{*}(X)$, endowed with the boundary operator $\partial$ extended linearly to $\mathcal{X} C_{*}(X)$, is a chain complex [3, Sect. 6.3]. When $X$ is a $G$-bornological coarse space, we let $\mathcal{X} C_{n}^{G}(X)$ be the subgroup of $\mathcal{X} C_{n}(X)$ given by the locally finite controlled $n$-chains that are also $G$-invariant. The boundary operator restricts to $\mathcal{X} C_{*}^{G}(X)$, and $\left(\mathcal{X} C_{*}^{G}(X), \partial\right)$ is a subcomplex of $\left(\mathcal{X} C_{*}(X), \partial\right)$.

If $f: X \rightarrow Y$ is a morphism of $G$-bornological coarse spaces, then we consider the map on the products $X^{n+1} \rightarrow Y^{n+1}$ sending $\left(x_{0}, \ldots, x_{n}\right)$ to $\left(f\left(x_{0}\right), \ldots, f\left(x_{n}\right)\right)$. It extends linearly to a map $\mathcal{X} C^{G}(f): \mathcal{X} C_{n}^{G}(X) \rightarrow \mathcal{X} C_{n}^{G}(Y)$ that involves sums over the pre-images by $f$. This describes a functor $\mathcal{X} C^{G}: G$ BornCoarse $\rightarrow$ Ch with values in the category $\mathbf{C h}$ of chain complexes over the integers. The $\infty$-category $\mathbf{C h}_{\infty}$ of chain complexes is defined as the localization (in the realm of $\infty$-categories [25, Sect. 1.3.4]) of the nerve of the category $\mathbf{C h}$ at the class $W$ of quasi-isomorphisms of chain complexes $\mathbf{C h}_{\infty}:=\mathrm{N}(\mathbf{C h})\left[W^{-1}\right]$. By post-composing the functor $\mathcal{X} C^{G}$ with the functor

$$
\mathcal{E M}: \mathbf{C h} \stackrel{\text { loc }}{\longrightarrow} \mathbf{C h}_{\infty} \stackrel{\simeq}{\rightarrow} H \mathbb{Z}-\mathbf{M o d} \rightarrow \mathbf{S p}
$$

(the Eilenberg-MacLane correspondence between chain complexes and spectra [33, Theorem 1.1] or [3, Sect. 6.3]), we get a functor to the $\infty$-category of spectra

$$
\mathcal{X} \mathrm{H}^{G}:=\mathcal{E} \mathcal{M} \circ \mathcal{X} C^{G}: G \text { BornCoarse } \rightarrow \mathbf{S p}
$$

called equivariant coarse ordinary homology:

Theorem $1.7\left[4\right.$, Theorem 7.3] The functor $\mathcal{X} \mathrm{H}^{G}$ is a $G$-equivariant $\mathbf{S p}$-valued coarse homology theory.

Example 1.8 If $X$ is a point, its coarse homology groups are 0 in positive and negative degree and the base ring $k$ in degree 0 .

\subsection{The category $V_{A}^{G}(X)$ of controlled objects}

The goal of this subsection is to recall the definition of the additive category $V_{\mathbf{A}}^{G}(X)$ of $G$-equivariant $X$-controlled A-objects [4, Definition 8.3] and of the functor $V_{\mathbf{A}}^{G}: G$ BornCoarse $\rightarrow$ Add sending a $G$-bornological coarse space to the category $V_{\mathbf{A}}^{G}(X)$. This functor is an essential ingredient in the construction of coarse homology theories like coarse algebraic $K$-homology and coarse Hochschild and cyclic homology.

Let $G$ be a group and let $X$ be a $G$-bornological coarse space.

Remark 1.9 The bornology $\mathcal{B}(X)$ on $X$ defines a poset with the partial order induced by subset inclusion; hence, $\mathcal{B}(X)$ can be seen as a category. 
Let $\mathbf{A}$ be an additive category with strict $G$-action. For every element $g$ in $G$ and every functor $F: \mathcal{B}(X) \rightarrow \mathbf{A}$, let $g F: \mathcal{B}(X) \rightarrow \mathbf{A}$ denote the functor sending a bounded set $B$ in $\mathcal{B}(X)$ to the A-object $g\left(F\left(g^{-1}(B)\right)\right)$ (and defined on morphisms $B \subseteq$ $B^{\prime}$ as the induced morphism of $\left.\mathbf{A}(g F)\left(B \subseteq B^{\prime}\right): g F\left(g^{-1}(B)\right) \rightarrow g F\left(g^{-1}\left(B^{\prime}\right)\right)\right)$.

If $\eta: F \rightarrow F^{\prime}$ is a natural transformation between two functors $F, F^{\prime}: \mathcal{B}(X) \rightarrow \mathbf{A}$, we denote by $g \eta: g F \rightarrow g F^{\prime}$ the induced natural transformation between $g F$ and $g F^{\prime}$.

Definition 1.10 [4, Definition 8.3] A G-equivariant $X$-controlled A-object is a pair $(M, \rho)$ consisting of a functor $M: \mathcal{B}(X) \rightarrow \mathbf{A}$ and a family $\rho=(\rho(g))_{g \in G}$ of natural isomorphisms $\rho(g): M \rightarrow g M$, satisfying the following conditions:

(1) $M(\emptyset) \cong 0$

(2) for all $B, B^{\prime}$ in $\mathcal{B}(X)$, the commutative diagram

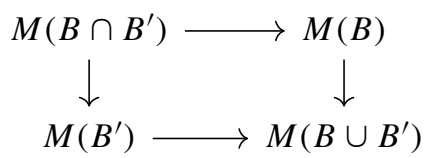

is a push-out;

(3) for all $B$ in $\mathcal{B}(X)$ there exists a finite subset $F$ of $B$ such that the inclusion induces an isomorphism $M(F) \stackrel{\cong}{\rightarrow} M(B)$;

(4) for all elements $g, g^{\prime}$ in $G$ we have the relation $\rho\left(g g^{\prime}\right)=g \rho\left(g^{\prime}\right) \circ \rho(g)$, where $g \rho\left(g^{\prime}\right)$ is the natural transformation from $g M$ to $g g^{\prime} M$ induced by $\rho\left(g^{\prime}\right)$.

Notation 1.11 If $(M, \rho)$ is an $X$-controlled $\mathbf{A}$-object and $x$ is an element of $X$, we will often write $M(x)$ instead of $M(\{x\})$ for the value of the functor $M$ at the bounded set $\{x\}$ of $X$.

Let $X$ be a $G$-bornological coarse space and let $(M, \rho)$ be an equivariant $X$ controlled A-object. Let $B$ be a bounded set of $X$ and let $x$ be a point in $B$. The inclusion $\{x\} \rightarrow B$ induces a morphism $M(\{x\}) \rightarrow M(B)$ of $\mathbf{A}$. The conditions of Definition 1.10 imply that $M(\{x\})=0$ for all but finitely many points of $B$ and that the canonical morphism (induced by the universal property of the coproduct in $\mathbf{A}$ )

$$
\bigoplus_{x \in B} M(\{x\}) \stackrel{\cong}{\rightarrow} M(B)
$$

is an isomorphism. The $U$-thickening $U[B]$ (1.1) of a bounded subset $B$ of $X$ is bounded and $U$-thickenings preserve the inclusions of bounded sets; we get a functor $U[-]: \mathcal{B}(X) \rightarrow \mathcal{B}(X)$.

Definition 1.12 [4, Definition 8.6] Let $(M, \rho)$ and $\left(M^{\prime}, \rho^{\prime}\right)$ be $G$-equivariant $X$ controlled A-objects and let $U \in \mathcal{C}^{G}(X)$ be a $G$-invariant entourage of $X$. A 
$G$-equivariant $U$-controlled morphism $\varphi:(M, \rho) \rightarrow\left(M^{\prime}, \rho^{\prime}\right)$ is a natural transformation

$$
\varphi: M(-) \rightarrow M^{\prime} \circ U[-]
$$

such that $\rho^{\prime}(g) \circ \varphi=(g \varphi) \circ \rho(g)$ for all $g$ in $G$.

The set of $G$-equivariant $U$-controlled morphisms $\varphi:(M, \rho) \rightarrow\left(M^{\prime}, \rho^{\prime}\right)$ is denoted by $\operatorname{Mor}_{U}\left((M, \rho),\left(M^{\prime}, \rho^{\prime}\right)\right)$. For every bounded set $B$ of $X$, the inclusion $U \subseteq U^{\prime}$ induces an inclusion $U[B] \subseteq U^{\prime}[B]$; this yields a natural transformation of functors $M^{\prime} \circ U[-] \rightarrow M^{\prime} \circ U^{\prime}[-]$, hence a map

$$
\operatorname{Mor}_{U}\left((M, \rho),\left(M^{\prime}, \rho^{\prime}\right)\right) \rightarrow \operatorname{Mor}_{U^{\prime}}\left((M, \rho),\left(M^{\prime}, \rho^{\prime}\right)\right)
$$

by post-composition.

By using these structure maps we define the abelian group of $G$-equivariant controlled morphisms from $(M, \rho)$ to $\left(M^{\prime}, \rho^{\prime}\right)$ as the colimit

$$
\operatorname{Hom}_{V_{\mathbf{A}}^{G}(X)}\left((M, \rho),\left(M^{\prime}, \rho^{\prime}\right)\right):=\operatorname{colim}_{U \in \mathcal{C}^{G}} \operatorname{Mor}_{U}\left((M, \rho),\left(M^{\prime}, \rho^{\prime}\right)\right) .
$$

Definition 1.13 [4] Let $X$ be a $G$-bornological coarse space and let $\mathbf{A}$ be an additive category with strict $G$-action. The category $V_{\mathbf{A}}^{G}(X)$ is the category of $G$-equivariant $X$-controlled A-objects and $G$-equivariant controlled morphisms.

Let $k$ be a field. When $\mathbf{A}$ is the category of finite-dimensional $k$-vector spaces, then we denote by $V_{k}^{G}(X)$ the associated category of $G$-equivariant $X$-controlled (finitedimensional) $k$-modules.

Lemma 1.14 [4, Lemma 8.7] The category of equivariant $X$-controlled A-objects $V_{\mathbf{A}}^{G}(X)$ is additive.

Let $f:(X, \mathcal{C}, \mathcal{B}) \rightarrow\left(X^{\prime}, \mathcal{C}^{\prime}, \mathcal{B}^{\prime}\right)$ be a morphism of $G$-bornological coarse spaces. If $(M, \rho)$ is a $G$-equivariant $X$-controlled $\mathbf{A}$-object, we consider the functor $f_{*} M: \mathcal{B}^{\prime} \rightarrow$ A defined by $f_{*} M\left(B^{\prime}\right):=M\left(f^{-1}\left(B^{\prime}\right)\right)$ for every bounded set $B^{\prime}$ in $\mathcal{B}^{\prime}$ and defined on morphisms in the canonical way. For every $g$ in $G$, the family of transformations $f_{*} \rho=$ $\left(\left(f_{*} \rho\right)(g)\right)_{g \in G}$ is given by the natural isomorphisms $\left(f_{*} \rho\right)(g): f_{*} M \rightarrow g\left(f_{*} M\right)$ with $\left(\left(f_{*} \rho\right)(g)\right)\left(B^{\prime}\right):=\rho(g)\left(f^{-1}\left(B^{\prime}\right)\right)$. The pair $f_{*}(M, \rho):=\left(f_{*} M, f_{*} \rho\right)$ defined in this way is a $G$-equivariant $X^{\prime}$-controlled A-object [4, Sect. 8.2]. Assume also that $U$ is an invariant entourage of $X$ and that $\varphi:(M, \rho) \rightarrow\left(M^{\prime}, \rho^{\prime}\right)$ is an equivariant $U$ controlled morphism. Then, the set $V:=(f \times f)(U)$ is a $G$-invariant entourage of $X^{\prime}$ and the morphism:

$$
f_{*} \varphi:=\left(f_{*} M\left(B^{\prime}\right) \stackrel{\varphi_{f^{-1}\left(B^{\prime}\right)}^{\longrightarrow}}{\longrightarrow} M\left(U\left[f^{-1}\left(B^{\prime}\right)\right]\right) \rightarrow f_{*} M\left(V\left[B^{\prime}\right]\right)\right)_{B^{\prime} \in \mathcal{B}^{\prime}}
$$

is $V$-controlled. We have just described a functor $f_{*}:=V_{\mathbf{A}}^{G}(f): V_{\mathbf{A}}^{G}(X) \rightarrow V_{\mathbf{A}}^{G}\left(X^{\prime}\right)$. 
We denote by

$$
V_{\mathbf{A}}^{G}: G \text { BornCoarse } \rightarrow \text { Add }
$$

the functor from the category of $G$-bornological coarse spaces to the category of small additive categories obtained in this way.

Remark 1.15 If $\mathbf{A}$ is a $k$-linear category, then the functor $V_{\mathbf{A}}^{G}: G$ BornCoarse $\rightarrow$ Add refines to a functor $V_{\mathbf{A}}^{G}: G$ BornCoarse $\rightarrow \mathbf{C a t}_{k}$ from the category of $G$-bornological coarse spaces to the category of small $k$-linear categories.

The following properties of the functor $V_{\mathbf{A}}^{G}$ are shown in [4]:

Remark 1.16 Let $(X, \mathcal{C}, \mathcal{B})$ be a $G$-bornological coarse space, $U \in \mathcal{C}^{G}$ a $G$-invariant entourage of $X$ and $X_{U}:=\left(X, \mathcal{C}_{U}, \mathcal{B}\right)$ the $G$-bornological coarse space obtained by restriction of the structures. Then, the category $V_{\mathbf{A}}^{G}(X)$ is the filtered colimit

$$
V_{\mathbf{A}}^{G}(X) \cong \operatorname{colim}_{U \in \mathcal{C}^{G}} V_{\mathbf{A}}^{G}\left(X_{U}\right)
$$

indexed on the poset of $G$-invariant entourages of $X$.

Lemma 1.17 [4, Lemma 8.11] Let $f, g: X \rightarrow X^{\prime}$ be two morphisms of $G$ bornological coarse spaces. If $f$ and $g$ are close to each other, then they induce naturally isomorphic functors $f_{*} \cong g_{*}: V_{\mathbf{A}}^{G}(X) \rightarrow V_{\mathbf{A}}^{G}\left(X^{\prime}\right)$.

Let $\mathcal{A}$ be an additive category and denote by $\oplus$ its biproduct. Recall that $\mathcal{A}$ is called flasque if it admits an endofunctor $S: \mathcal{A} \rightarrow \mathcal{A}$ and a natural isomorphism id $\mathcal{A} \oplus S \cong S$.

Lemma 1.18 [4, Lemma 8.13] If $X$ is a flasque G-bornological coarse space, then the category $V_{\mathbf{A}}^{G}(X)$ of $G$-equivariant $X$-controlled $\mathbf{A}$-objects is a flasque category.

We conclude with the definition of coarse algebraic $K$-homology:

Definition 1.19 [4, Definition 8.8] Let $G$ be a group and let $\mathbf{A}$ be an additive category with strict $G$-action. The $G$-equivariant coarse algebraic $K$-homology associated to $\mathbf{A}$ is the $K$-theory of the additive category of A-controlled objects:

$$
K \mathbf{A} \mathcal{X}^{G}:=K \circ V_{\mathbf{A}}^{G}: G \text { BornCoarse } \rightarrow \text { Sp. }
$$

When $\mathbf{A}$ is the category of finite-dimensional $k$-vector spaces, we denote by $K \mathcal{X}_{k}^{G}$ the associated $K$-theory functor. The properties of the functor $V_{\mathbf{A}}^{G}$ reviewed above are used in order to prove the following:

Theorem 1.20 [4, Theorem 8.9] Let $G$ be a group and let $\mathbf{A}$ be an additive category with strict $G$-action. Then, the functor $K \mathbf{A} \mathcal{X}^{G}$ is a $G$-equivariant $\mathbf{S p - v a l u e d ~ c o a r s e}$ homology theory. 


\section{Keller's cyclic homology for dg-categories}

In this section we recall Keller's construction of cyclic homology for dg-categories [21]. We start by recalling some properties of differential graded categories and mixed complexes, we introduce Keller's construction and then review Keller's Localization Theorem [21, Theorem 1.5]. Keller defines the cyclic homology of a dg-category as the cyclic homology of a suitable mixed complex associated to it. We point here that, in the next Sect. 3 and in particular in Definition 3.1, we will need Keller's cyclic homology in the less general context of additive ( $k$-linear) categories. However, for consistency with his language and for sake of completeness, we will state Keller's definition and results in the broader context of dg-categories.

\subsection{Dg-categories}

In the following, we use the same conventions on differential graded categories and their properties as found in [20]; we refer to the same survey for a general overview on the subject. We recall that a dg-category over $k$ is a category enriched on (the category of) chain complexes of $k$-modules and that every additive, or $k$-linear, category, is a dgcategory in a canonical way. We denote by dgcat $_{k}$ the category of small dg-categories (over $k$ ) and dg-functors.

Remark 2.1 The category of dg-modules (over a dg-algebra or a dg-category) admits two Quillen model structures where the weak equivalences are the objectwise quasiisomorphisms of dg-modules; these are the injective and the projective model structure induced from the injective and projective model structure on chain complexes, respectively. We remark that the category of dg-modules over a dg-algebra, equipped with the projective model structure (hence the fibrations are the objectwise epimorphisms), is a combinatorial model category; see, for example, [10, Remark 2.14].

If $\mathcal{A}$ is a dg-category, we can define an associated derived category:

Definition 2.2 [20, Sect.3.2] The derived category $\mathcal{D}(\mathcal{A})$ of a dg-category $\mathcal{A}$ is the localization of the category of $\operatorname{dg}$-modules over $\mathcal{A}$ at the class of quasi-isomorphisms.

The objects of $\mathcal{D}(\mathcal{A})$ are the $\operatorname{dg}$-modules over $\mathcal{A}$ and the morphisms are obtained from morphisms of dg-modules by inverting the quasi-isomorphisms. It is a triangulated category with shift functor induced by the 1-translation and triangles coming from short exact sequences of complexes.

Let $\mathcal{A}$ and $\mathcal{B}$ be two small dg-categories. A dg-functor $F: \mathcal{A} \rightarrow \mathcal{B}$ is called a Morita equivalence if it induces an equivalence of derived categories. For a precise definition of Morita equivalences we refer to [20, Sect. 3.8], or [10, Definition 2.29].

Theorem 2.3 [34, Theorem 5.1] The category dgcat $_{k}$ of small dg-categories over $k$ admits the structure of a combinatorial model category whose weak equivalences are the Morita equivalences.

For a description of fibrations and cofibrations we refer to [34, Theorem 5.1], or [20, Theorem 4.1]. We conclude with the definition of short exact sequences of dgcategories: 
Definition 2.4 [20, Sect. 4.6] A short exact sequence of dg-categories is a sequence of morphisms $\mathcal{A} \rightarrow \mathcal{B} \rightarrow \mathcal{C}$ inducing an exact sequence of triangulated categories

$$
\mathcal{D}^{b}(\mathcal{A}) \rightarrow \mathcal{D}^{b}(\mathcal{B}) \rightarrow \mathcal{D}^{b}(\mathcal{C})
$$

in the sense of Verdier.

\subsection{The $\infty$-category of mixed complexes}

In this subsection we describe the (cocomplete stable $\infty$-)category of unbounded mixed complexes. We follow Kassel's approach [19].

Definition 2.5 [19, Sect. 1] A mixed complex $(C, b, B)$ is a triple consisting of a $\mathbb{Z}$-graded $k$-module $C=\left(C_{p}\right)_{p \in \mathbb{Z}}$ together with differentials $b$ and $B$

$$
b=\left(b_{p}: C_{p} \rightarrow C_{p-1}\right)_{p \in \mathbb{Z}} \quad \text { and } \quad B=\left(B_{p}: C_{p} \rightarrow C_{p+1}\right)_{p \in \mathbb{Z}}
$$

of degree -1 and 1 , respectively, satisfying the following identities:

$$
b^{2}=0, \quad B^{2}=0, \quad b B+B b=0 .
$$

Morphisms of mixed complexes are given by maps commuting with both the differentials $b$ and $B$. The category of mixed complexes and morphisms of mixed complexes is denoted by Mix.

When the differentials are clear from the context, we refer to a mixed complex $(C, b, B)$ by its underlying $k$-module $C$.

Let $\Lambda$ be the dg-algebra over the field $k$

$$
\Lambda:=\cdots \rightarrow 0 \rightarrow k \epsilon \stackrel{0}{\rightarrow} k \rightarrow 0 \rightarrow \cdots
$$

generated by an indeterminate $\epsilon$ of degree 1, with $\epsilon^{2}=0$ and differential (of degree $-1) d(\epsilon)=0$. Mixed complexes are nothing but dg-modules over the dg-algebra $\Lambda$ :

Remark 2.6 [19] The category Mix of mixed complexes is equivalent (in fact, isomorphic) to the category of left $\Lambda$-dg-modules, which we denote by $\Lambda$-Mod. We denote by $L:$ Mix $\rightarrow \Lambda$-Mod the functor sending a mixed complex to the associated $\Lambda$-dg-module and by $R: \Lambda$-Mod $\rightarrow$ Mix its inverse functor.

The category of $k$-dg-modules admits a combinatorial model structure (the projective model structure, see Remark 2.1), whose weak equivalences are the objectwise quasi-isomorphisms of dg-modules. In the language of mixed complexes this translates as follows:

Definition 2.7 A morphism $(C, b, B) \rightarrow\left(C^{\prime}, b^{\prime}, B^{\prime}\right)$ of mixed complexes is called a quasi-isomorphism if the underlying $b$-complexes are quasi-isomorphic via the induced chain map $(C, b) \rightarrow\left(C^{\prime}, b^{\prime}\right)$. 
Remark 2.8 Quasi-isomorphisms of mixed complexes correspond to quasiisomorphisms of $\Lambda$-dg-modules, i.e., the functors $L$ and $R$ of Remark 2.6 preserve quasi-isomorphisms.

If $\mathbf{C}$ is an ordinary category and $W$ denotes a collection of morphisms of $\mathbf{C}$, then $\mathrm{N}(\mathbf{C})\left[W^{-1}\right]$ is the $\infty$-category obtained by the nerve $\mathrm{N}(\mathbf{C})$ of $\mathbf{C}$ by inverting the set of morphisms $W$ (see [9, Definition 7.1.2 and Proposition 7.1.3], [25, Definition 1.3.4.1]).

Definition 2.9 The $\infty$-category

$$
\mathbf{M i x}_{\infty}:=\mathrm{N}(\mathbf{M i x})\left[W_{\text {mix }}^{-1}\right]
$$

of mixed complexes is defined as the localization of the (nerve of the) category Mix at the class $W_{\text {mix }}$ of quasi-isomorphisms of mixed complexes.

Analogously, the $\infty$-category $\Lambda$-Mod $\infty$ is defined as the localization of the category $\Lambda$-Mod of $\Lambda$-dg-modules at the class $W$ of quasi-isomorphisms of $\Lambda$-dg-modules:

$$
\Lambda-\operatorname{Mod}_{\infty}:=\mathrm{N}(\Lambda-\operatorname{Mod})\left[W^{-1}\right]
$$

Proposition 2.10 The $\infty$-category $\mathbf{M i x _ { \infty }}$ is a cocomplete stable $\infty$-category.

Proof The category $\Lambda$-Mod is a (pre-triangulated) dg-category. By applying the dgnerve functor $\mathrm{N}_{\mathrm{dg}}$ [25, Constr. 1.3.1.6] we obtain an $\infty$-category $\mathrm{N}_{\mathrm{dg}}(\Lambda$-Mod) [25, Proposition 1.3.1.10]. The dg-nerve functor sends pre-triangulated dg-categories to stable $\infty$-categories [14, Theorem 4.3.1], [25, Proposition 1.3.1.10]. The $\infty$-category $\mathrm{N}_{\mathrm{dg}}(\Lambda$-Mod) is stable and its homotopy category can be identified (as a triangulated category) with the derived category $\mathcal{D}(\Lambda)$ associated to the dg-algebra $\Lambda$.

The category $\Lambda$-Mod is equipped with a combinatorial simplicial model structure by Remark 2.1. By [25, Proposition 1.3.1.17] and by the fact that the simplicial nerve of the simplicial category associated to $\Lambda$-Mod is equivalent to the localization $\mathrm{N}\left(\Lambda\right.$-Mod) $\left[W^{-1}\right]$ (by [25, Remark 1.3.4.16 and Thm. 1.3.4.20] where we also use that the model category $\Lambda$-Mod is combinatorial, hence admits functorial factorizations), the two constructions $\mathrm{N}(\Lambda$-Mod $)\left[W^{-1}\right]$ and $\mathrm{N}_{\mathrm{dg}}(\Lambda$-Mod $)$ present equivalent $\infty$-categories. Hence, the $\infty$-category $\Lambda-\operatorname{Mod}_{\infty}$ is a stable $\infty$-category. The $\infty$ category $\Lambda-\mathbf{M o d}_{\infty}$ is also cocomplete by [25, Proposition 1.3.4.22] because the model category $\Lambda$-Mod is combinatorial.

The categories Mix and $\Lambda$-Mod are isomorphic by Remark 2.6 and the functor $L:$ Mix $\rightarrow \Lambda$-Mod and its inverse $R: \Lambda$-Mod $\rightarrow$ Mix preserve quasi-isomorphisms by Remark 2.8 . This yields an equivalence of $\infty$-categories

$$
\mathrm{N}(\mathbf{M i x})\left[W_{\text {mix }}^{-1}\right] \rightarrow \mathrm{N}(\Lambda \text {-Mod })\left[W^{-1}\right]
$$

that proves the statement.

Remark 2.11 The homotopy category of the stable $\infty$-category $\mathbf{M i x} \mathbf{x}_{\infty}$ is canonically equivalent to the derived category $\mathcal{D}(\Lambda)$ of the dg-algebra $\Lambda$. 
We conclude the subsection with the definition of Hochschild and cyclic homology of mixed complexes. A mixed complex $(C, b, B)$ functorially determines a double chain complex $\mathcal{B} C$ [22, Sect. 2.5.10] by means of the differentials $b$ and $B$ :

$$
\mathcal{B C}:=\left(\cdots \stackrel{0}{\leftarrow}(C, b) \stackrel{B}{\leftarrow}\left(C[-1], b_{C[-1]}\right) \stackrel{B}{\leftarrow} \cdots \stackrel{B}{\leftarrow}\left(C[-n], b_{C[-n]}\right) \stackrel{B}{\leftarrow} \cdots\right) ;
$$

here, the chain complex $(C, b)$ is placed in bi-degree $(0, *)$, i.e., $\mathcal{B} C_{(0, *)}=\left(C_{*}, b\right)$, and the chain complex $\left(C[-n], b_{C[-n]}\right)$, placed in bi-degree $(n, *)$, is the chain complex $(C, b)$ shifted by $-n$, hence $\mathcal{B} C_{(p, q)}=C_{q-p}$ for $p \geq 0$ and $\mathcal{B} C_{(p, q)}=0$ for $p<0$. The total chain complex $\operatorname{Tot}(\mathcal{B C})$, functorially associated to the double chain complex $\mathcal{B} C$, is the chain complex defined in degree $n$ by $\operatorname{Tot}_{n}(\mathcal{B C})=\bigoplus_{i \geq 0} C_{n-2 i}$ with differential $d$ acting as follows: $d\left(c_{n}, c_{n-2}, \ldots\right):=\left(b c_{n}+B c_{n-2}, \ldots\right)$.

Let $\mathbf{C h}$ be the category of chain complexes over $k$. Consider the forgetful functor

$$
\text { forget: } \mathbf{M i x} \rightarrow \mathbf{C h}
$$

sending a mixed complex $(M, b, B)$ to its underlying chain complex $(M, b)$, and the functor

$$
\operatorname{Tot}(\mathcal{B}-): \mathbf{M i x} \rightarrow \mathbf{C h}
$$

just described above.

Definition 2.12 [19, Sect. 1] Let $(C, b, B)$ be a mixed complex. The Hochschild homology $\mathrm{HH}_{*}(C)$ of $(C, b, B)$ is the homology of the underlying chain complex $(C, b)$. Its cyclic homology $\mathrm{HC}_{*}(C)$ is the homology of the associated chain complex $\operatorname{Tot}(\mathcal{B C})$.

We remark that this definition agrees with the classical definition of Hochschild and cyclic homology of algebras [19,22].

\subsection{Keller's cyclic homology}

Let $k$ be a commutative ring with identity and let $A$ be a $k$-algebra. Then, one can associate to $A$ a cyclic module $Z_{*}(A)$ [15] (i.e., a cyclic object in the category of $k$-modules) defined in degree $n$ as the $(n+1)$-th tensor product of $A$ over $k$. In the same way, one can construct a cyclic module out of an additive category $\mathcal{A}[26$, Definition 2.1.1]. We present these constructions in the more general setting of dgcategories.

Definition 2.13 [21] Let $\mathcal{C}$ be a small dg-category over $k$. The additive cyclic nerve of $\mathcal{C}$ is the cyclic $k$-module defined by:

$$
\mathrm{CN}_{n}(\mathcal{C}):=\bigoplus \operatorname{Hom}_{\mathcal{C}}\left(C_{1}, C_{0}\right) \otimes \operatorname{Hom}_{\mathcal{C}}\left(C_{2}, C_{1}\right) \otimes \cdots \otimes \operatorname{Hom}_{\mathcal{C}}\left(C_{0}, C_{n}\right)
$$


where the sum runs over all the objects $\left(C_{0}, C_{1}, \ldots, C_{n}\right)$ in $\mathcal{C}^{n+1}$. The face and degeneracy maps, and the cyclic action, are defined as follows:

$$
\begin{aligned}
& d_{i}\left(f_{0} \otimes \cdots \otimes f_{n}\right)= \begin{cases}f_{0} \otimes f_{1} \otimes \cdots \otimes f_{i} \circ f_{i+1} \otimes \cdots \otimes f_{n} & \text { if } 0 \leq i \leq n-1, \\
(-1)^{n+\sigma} f_{n} \circ f_{0} \otimes f_{1} \otimes \cdots \otimes f_{n-1} & \text { if } i=n,\end{cases} \\
& s_{i}\left(f_{0} \otimes \cdots \otimes f_{n}\right)= \begin{cases}f_{0} \otimes f_{1} \otimes \cdots \otimes f_{i} \otimes \operatorname{id}_{C_{i+1}} \otimes f_{i+1} \otimes \cdots \otimes f_{n} & \text { if } 0 \leq i \leq n-1, \\
f_{0} \otimes f_{1} \otimes \cdots \otimes f_{n} \otimes \operatorname{id}_{C_{0}} & \text { if } i=n,\end{cases} \\
& t\left(f_{0} \otimes \cdots \otimes f_{n}\right)=(-1)^{n+\sigma}\left(f_{n} \otimes f_{0} \otimes \cdots \otimes f_{n-1}\right),
\end{aligned}
$$

where $\sigma=\left(\operatorname{deg} f_{n}\right)\left(\operatorname{deg} f_{n-1}+\cdots+\operatorname{deg} f_{0}\right)$.

We get a covariant functor from the category of small dg-categories over $k$ to the category of cyclic $k$-modules. To every cyclic $k$-module $M$, we can further associate a mixed complex by letting $b: M_{n} \rightarrow M_{n-1}$ be the alternating sum

$$
b:=\sum_{i=0}^{n}(-1)^{i} d_{i}
$$

of face maps, and by defining the cochain map $B: M_{n} \rightarrow M_{n+1}$ as the composition

$$
B:=(-1)^{n+1}\left(1-t_{n+1}\right) s N
$$

Here, $s$ denotes the extra degeneracy $s=(-1)^{n+1} t_{n+1} s_{n}: M_{n} \rightarrow M_{n+1}$ and $N:=$ $\sum_{i=0}^{n} t_{n+1}^{i}$.

Remark 2.14 Let $M$ be a cyclic module. Then, the triple $(M, b, B)$, where $b$ and $B$ are the differentials (2.6) and (2.7), respectively, is a mixed complex. Morphisms of cyclic modules commute with the face and the degeneracy maps and with the cyclic operators; they yield in this way morphisms of mixed complexes and a functor from the category of cyclic modules to the category of mixed complexes.

Definition 2.15 [21, Definition 1.3] We denote by

$$
\text { Mix : } \text { dgcat }_{k} \rightarrow \text { Mix }
$$

the functor from the category of small dg-categories over $k$ to the category of mixed complexes defined as composition of the additive cyclic nerve functor of Definition 2.13 with the functor of Remark 2.14.

Thanks to the work of Keller, we know that this functor enjoys many useful properties, among others agreement, additivity and localization [21]. As we work in the context of $\infty$-categories, we will spell them out in this language.

From now on we assume that $k$ is a field. The $\infty$-category of small dg-categories $\operatorname{dgcat}_{k, \infty}:=\mathrm{N}\left(\right.$ dgcat $\left._{k}\right)\left[W_{\text {Morita }}^{-1}\right]$ is the localization at the class $W_{\text {Morita }}$ of Morita equivalences. By [21, Theorem 1.5], the functor Mix of Definition 2.15 sends 
Morita equivalences of dg-categories to quasi-isomorphisms of mixed complexes and descends to a functor

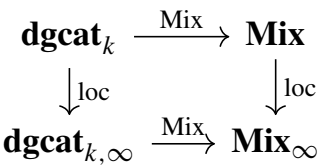

between the localizations. Keller's Localization Theorem [21, Theorem 1.5] can then be summarized as follows:

Theorem 2.16 [21, Theorem 1.5] Let $k$ be a field. The functor Mix: $\mathbf{d g c a t}_{k} \rightarrow \mathbf{M i x}_{\infty}$ satisfies the following:

(1) it sends equivalences of small dg-categories to equivalences of mixed complexes;

(2) it commutes with filtered colimits;

(3) it sends short exact sequences $\mathcal{A} \rightarrow \mathcal{B} \rightarrow \mathcal{C}$ ofdg-categories to cofiber sequences of $\mathbf{M i x}_{\infty}$.

Moreover, if $A$ is a $k$-algebra, there is an equivalence of mixed complexes

$$
\operatorname{Mix}(A) \rightarrow \operatorname{Mix}(\operatorname{proj} A)
$$

where $\operatorname{proj} A$ is the additive category of finitely generated projective modules.

Observe that the functor Mix o loc preserves filtered colimits. By Proposition 2.10, the $\infty$-category $\mathbf{M i x} \mathbf{x}_{\infty}$ is stable and cocomplete and cofiber sequences of $\mathbf{M i x} \mathbf{x}_{\infty}[25$, Definition 1.1.1.6] are detected in its homotopy category, i.e., in $\mathcal{D}(\Lambda)$. We observe here that Keller's theorem holds in a more general setting (for more general rings and for exact categories). However, we only need these properties in the context of additive categories (over a field). Moreover, in this context, Keller's functor Mix is equivalent to the cyclic homology functor constructed by McCarthy [26] (see also [8, Lemma 3.4.4 and Remark 3.4.5]).

\section{Equivariant coarse Hochschild and cyclic homology}

For a fixed base field $k$ and group $G$, we define equivariant coarse Hochschild $\mathcal{X} \mathrm{HH}_{k}^{G}$ and cyclic homology $\mathcal{X} \mathrm{HC}_{k}^{G}$ versions of the classical Hochschild and cyclic homology of $k$-algebras. This is achieved by first studying an intermediate equivariant coarse homology theory $\mathcal{X} \mathrm{Mix}_{k}^{G}$ with values in the $\infty$-category of mixed complexes. In the definition of $\mathcal{X} \mathrm{Mix}_{k}^{G}$, we employ Keller's functor Mix: $\operatorname{dgcat}_{k} \rightarrow$ Mix in the context of $k$-linear categories, and we apply it to the category $V_{k}^{G}(X)$ of controlled objects; we then define Hochschild/cyclic homology of a bornological coarse space $X$ as Hochschild/cyclic homology of $\operatorname{Mix}\left(V_{k}^{G}(X)\right)$. We conclude the section with some properties of these homology theories and of the associated assembly maps. 


\subsection{The equivariant coarse homology theory $\mathcal{X} \mathrm{Mix}_{k}^{G}$}

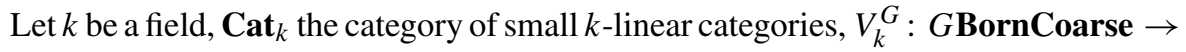
Cat $_{k}$ the functor of Remark 1.15, let Mix: dgcat $_{k} \rightarrow$ Mix be the functor of Definition 2.15, $\iota:$ Cat $_{k} \rightarrow$ dgcat $_{k}$ the functor sending a $k$-linear category to its associated dg-category and loc the localization functor loc: $\mathbf{M i x} \rightarrow \mathbf{M i x}_{\infty}$.

Definition 3.1 We denote by $\mathcal{X} \mathrm{Mix}_{k}^{G}$ the following functor

$$
\mathcal{X} \operatorname{Mix}_{k}^{G}: G \text { BornCoarse } \stackrel{V_{k}^{G}}{\longrightarrow} \text { Cat }_{k} \stackrel{\iota}{\longrightarrow} \text { dgcat }_{k} \stackrel{\text { Mix }}{\longrightarrow} \text { Mix } \stackrel{\text { loc }}{\longrightarrow} \text { Mix }_{\infty}
$$

from the category of $G$-bornological coarse spaces to the $\infty$-category of mixed complexes.

The proof that the functor $\mathcal{X} \mathrm{Mix}_{k}^{G}$ satisfies the axioms of Definition 1.5 describing an equivariant coarse homology theory follows the ideas of coarse algebraic $K$-homology [4, Sect. 8] and does not require assumptions on $G$ or $k$. For every $G$-set $X$ and additive category with $G$-action $\mathbf{A}$, the category $V_{\mathbf{A}}^{G}\left(X_{\min , \max } \otimes G_{\text {can, } \min }\right)$ is equivalent to the additive category $\mathbf{A} *_{G} X$ [7, Definition 2.1] by [4, Definition 8.21 and Proposition 8.24]. As a consequence, $\mathcal{X} \operatorname{Mix}_{k}^{G}\left(X_{\min , \max } \otimes G_{\mathrm{can}, \min }\right) \simeq \operatorname{Mix}\left(\mathbf{A} *_{G} X\right)$, and the functor $\mathcal{X} \mathrm{Mix}_{k}^{G}$, when applied to such spaces, can be described as the mixed complex of a suitable additive category. In the case of the group $G$, this result says that the category of controlled objects $V_{k}^{G}\left(G_{\text {can,min }}\right)$ is equivalent to the category of finitely generated free $k[G]$-modules (see Proposition 3.15), which, together with Proposition 3.14, further justifies the use of Definition 3.1 in the construction of a cyclic homology theory for $G$-bornological coarse spaces.

The main result of the section is the following theorem:

Theorem 3.2 The functor

$$
\mathcal{X} \operatorname{Mix}_{k}^{G}: G \text { BornCoarse } \longrightarrow \operatorname{Mix}_{\infty}
$$

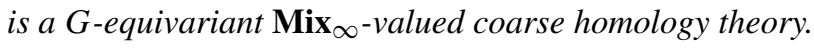

Proof The category $\mathbf{M i x} \mathbf{x}_{\infty}$ is stable and cocomplete by Proposition 2.10. We prove below that the functor $\mathcal{X} \mathrm{Mix}_{k}^{G}$ satisfies coarse invariance (see Proposition 3.3), vanishing on flasque spaces (see Proposition 3.4), u-continuity (see Proposition 3.5) and coarse excision (see Theorem 3.6), i.e., the axioms describing an equivariant coarse homology theory.

Proposition 3.3 The functor $\mathcal{X} \mathrm{Mix}_{k}^{G}:$ GBornCoarse $\rightarrow \mathbf{M i x}_{\infty}$ satisfies coarse invariance.

Proof If $f: X \rightarrow Y$ is a coarse equivalence of $G$-bornological coarse spaces, then it induces a natural equivalence $f_{*}: V_{k}^{G}(X) \rightarrow V_{k}^{G}(Y)$ by Lemma 1.17. Keller's functor Mix sends equivalences of dg-categories to equivalences of mixed complexes 
by Theorem $2.16(1)$. Hence, the functor $f_{*}$ induces an equivalence $\mathcal{X} \operatorname{Mix}_{k}^{G}(X) \stackrel{\sim}{\rightarrow}$ $\mathcal{X} \operatorname{Mix}_{k}^{G}(Y)$ in $\mathbf{M i x}{ }_{\infty}$, i.e., the functor $\mathcal{X} \operatorname{Mix}_{k}^{G}$ is coarse invariant.

Recall the definition of flasque spaces in Definition 1.3.

Proposition 3.4 The functor $\mathcal{X} \mathrm{Mix}_{k}^{G}:$ BBornCoarse $\rightarrow \mathbf{M i x}_{\infty}$ vanishes on flasque spaces.

Proof By Lemma 1.18, the category $V_{k}^{G}(X)$ is a flasque category, hence there exists an endofunctor $S: V_{k}^{G}(X) \rightarrow V_{k}^{G}(X)$ such that $\operatorname{id}_{V_{k}^{G}(X)} \oplus S \cong S$. By [32, Theorem 2.3.11] (see also [8, Theorem 3.3.5]), the morphisms $\operatorname{Mix}(i d) \oplus \operatorname{Mix}(S)$ and $\operatorname{Mix}(\operatorname{id} \oplus S) \cong \operatorname{Mix}(S)$ are equivalent in $\mathbf{M i x} \mathbf{x}_{\infty}$. This means that the morphism

$$
\mathcal{X} \operatorname{Mix}_{k}^{G}(\mathrm{id}): \mathcal{X} \operatorname{Mix}_{k}^{G}(X) \rightarrow \mathcal{X} \operatorname{Mix}_{k}^{G}(X)
$$

is equivalent to the 0 -morphism and that $\mathcal{X} \operatorname{Mix}_{k}^{G}(X) \simeq 0$.

Proposition 3.5 The functor $\mathcal{X} \mathrm{Mix}_{k}^{G}:$ BBornCoarse $\rightarrow \mathbf{M i x}_{\infty}$ is u-continuous.

Proof Let $X$ be a $G$-bornological coarse space, and let $\mathcal{C}^{G}$ be the poset of $G$ invariant controlled sets. By Remark 1.16, there is an equivalence $V_{k}^{G}(X) \simeq$ $\operatorname{colim}_{U \in \mathcal{C}^{G}} V_{k}^{G}\left(X_{U}\right)$ of $k$-linear categories, hence of dg-categories. The functor Mix commutes with filtered colimits, and we get the equivalence

$$
\mathcal{X M i x}_{k}^{G}(X) \simeq \operatorname{colim}_{U \in \mathcal{C}^{G}} \mathcal{X} \operatorname{Mix}_{k}^{G}\left(X_{U}\right)
$$

in $\mathbf{M i x} x_{\infty}$, which shows that the functor $\mathcal{X} \operatorname{Mix}_{k}^{G}$ is u-continuous.

Theorem 3.6 The functor $\mathcal{X} \mathrm{Mix}_{k}^{G}:$ GBornCoarse $\rightarrow$ Mix $\infty$ satisfies coarse excision.

Before giving the proof of this theorem we first need some more terminology.

Definition 3.7 [18] A full additive subcategory $\mathcal{A}$ of an additive category $\mathcal{U}$ is a Karoubi-filtration if every diagram $X \rightarrow Y \rightarrow Z$ in $\mathcal{U}$, with $X, Z \in \mathcal{A}$, admits an extension

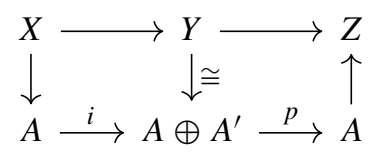

for some object $A \in \mathcal{A}$.

By [18, Lemma 5.6], this definition is equivalent to the classical one $[11,17]$. If $\mathcal{A}$ is a Karoubi-filtration of $\mathcal{U}$, we can construct a quotient category $\mathcal{U} / \mathcal{A}$. Its objects are the objects of $\mathcal{U}$, and the morphisms sets are defined as follows:

$$
\operatorname{Hom}_{\mathcal{U} / \mathcal{A}}(U, V):=\operatorname{Hom}_{\mathcal{U}}(U, V) / \sim
$$


where the relation identifies pairs of maps $U \rightarrow V$ whose difference factors through an object of $\mathcal{A}$.

Let $X$ be a $G$-bornological coarse space and let $\mathcal{Y}=\left(Y_{i}\right)_{i \in I}$ be an equivariant big family on $X$ (see Definition 1.4). The bornological coarse space $Y_{i}$ is a subspace of $X$ with the induced bornology and coarse structure. The inclusion $Y_{i} \hookrightarrow X$ induces a functor $V_{k}^{G}\left(Y_{i}\right) \rightarrow V_{k}^{G}(X)$ which is injective on objects. The categories $V_{k}^{G}\left(Y_{i}\right)$ and $V_{k}^{G}(\mathcal{Y}):=\operatorname{colim}_{i \in I} V_{k}^{G}\left(Y_{i}\right)$ are full subcategories of $V_{k}^{G}(X)$.

Lemma 3.8 [4, Lemma 8.14] Let $\mathcal{Y}$ be an equivariant big family on the G-bornological coarse space $X$. Then, the full additive subcategory $V_{k}^{G}(\mathcal{Y})$ of $V_{k}^{G}(X)$ is a Karoubi filtration.

Let $X$ be a $G$-bornological coarse space, and let $(Z, \mathcal{Y})$ be an equivariant complementary pair. Consider the functor

$$
a: V_{k}^{G}(Z) / V_{k}^{G}(Z \cap \mathcal{Y}) \rightarrow V_{k}^{G}(X) / V_{k}^{G}(\mathcal{Y})
$$

induced by the inclusion $i: Z \rightarrow X$; on objects, it coincides with $i_{*}: V_{k}^{G}(Z) \rightarrow$ $V_{k}^{G}(X)$, but on morphisms it sends the equivalence class [A] of $A$ to the equivalence class $\left[i_{*}(A)\right]$ of $i_{*}(A)$.

Lemma 3.9 [4, Proposition 8.15] The functor a in (3.1) is an equivalence of categories.

Proof of Theorem 3.6 Let $X$ be a $G$-bornological coarse space, and let $(Z, \mathcal{Y})$ be an equivariant complementary pair on $X$. By Lemma 3.8, $V_{k}^{G}(Z \cap \mathcal{Y}) \subseteq V_{k}^{G}(Z)$ and $V_{k}^{G}(\mathcal{Y}) \subseteq V_{k}^{G}(X)$ are Karoubi filtrations and yield the following sequences of $k$-linear categories:

$$
V_{k}^{G}(Z \cap \mathcal{Y}) \rightarrow V_{k}^{G}(Z) \rightarrow V_{k}^{G}(Z) / V_{k}^{G}(Z \cap \mathcal{Y})
$$

and

$$
V_{k}^{G}(\mathcal{Y}) \rightarrow V_{k}^{G}(X) \rightarrow V_{k}^{G}(X) / V_{k}^{G}(X \cap \mathcal{Y})
$$

By [31, Example. 1.8, Proposition 2.6] (see also [8, Remark 3.3.12]), Karoubi filtrations induce short exact sequences of dg-categories. Hence, Theorem 2.16 gives cofiber sequences of mixed complexes. The inclusion $Z \hookrightarrow X$ induces a commutative diagram (where the rows are the obtained cofiber sequences)

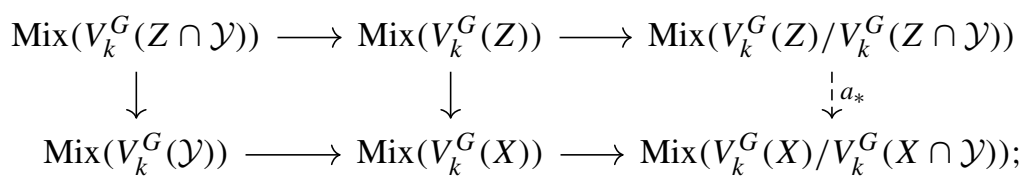

here $a_{*}$ is the map induced by $a: V_{k}^{G}(Z) / V_{k}^{G}(Z \cap \mathcal{Y}) \rightarrow V_{k}^{G}(X) / V_{k}^{G}(\mathcal{Y})$ in (3.1). By Lemma 3.9, the functor $a$ yields an equivalence of categories, hence of mixed complexes and the left square is a co-Cartesian square in $\mathbf{M i x} \mathbf{x}_{\infty}$. 
In order to conclude the proof, we recall that $\mathcal{X} \operatorname{Mix}_{k}^{G}(\mathcal{Y})$ is defined as the filtered colimit $\mathcal{X} \operatorname{Mix}_{k}^{G}(\mathcal{Y})=\operatorname{colim}_{i} \mathcal{X} \operatorname{Mix}_{k}^{G}\left(Y_{i}\right)$ and that $V_{k}^{G}(\mathcal{Y}):=\operatorname{colim}_{i \in I} V_{k}^{G}\left(Y_{i}\right)$. The functor Mix commutes with filtered colimits of dg-categories. Hence we have the equivalence $\operatorname{Mix}\left(V_{k}^{G}(\mathcal{Y})\right)=\operatorname{Mix}\left(\operatorname{colim}_{i} V_{k}^{G}\left(Y_{i}\right)\right) \simeq \operatorname{colim}_{i} \operatorname{Mix}\left(V_{k}^{G}\left(Y_{i}\right)\right)$, and the same holds for $Z \cap \mathcal{Y}$. By using these identifications, we obtain the co-Cartesian square in $\mathbf{M i x} \infty$

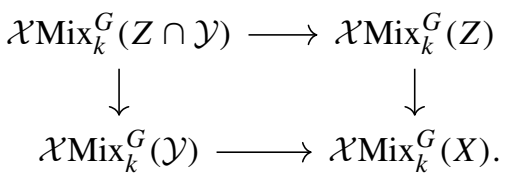

This means that $\mathcal{X} \mathrm{Mix}_{k}^{G}$ satisfies coarse excision.

\subsection{Coarse Hochschild and cyclic homology}

The functors forget: $\mathbf{M i x} \rightarrow \mathbf{C h}$ in (2.4), sending a mixed complex to the underlying chain complex, and $\operatorname{Tot}(\mathcal{B}-):$ Mix $\rightarrow \mathbf{C h}$ in (2.5), sending a mixed complex to the total complex of its associated bicomplex, send quasi-isomorphisms of mixed complexes to quasi-isomorphisms of chain complexes. Hence they descend to functors between the localizations.

Definition 3.10 Let $k$ be a field, $G$ a group and $\mathbf{C h}_{\infty}$ the $\infty$-category of chain complexes. The $G$-equivariant coarse Hochschild homology $\mathcal{X} \mathrm{HH}_{k}^{G}$ (with $k$-coefficients) is the $G$-equivariant $\mathbf{C h}_{\infty}$-valued coarse homology theory

$$
\mathcal{X} \mathrm{HH}_{k}^{G}: G \text { BornCoarse } \stackrel{\mathcal{X} \operatorname{Mix}_{k}^{G}}{\longrightarrow} \mathbf{M i x}_{\infty} \stackrel{\text { forget }}{\longrightarrow} \mathbf{C h}_{\infty}
$$

defined as composition of the functor $\mathcal{X} \mathrm{Mix}_{k}^{G}$ of Definition 3.1 and of the functor forget in (2.4). The composition

$$
\mathcal{X} \mathrm{HC}_{k}^{G}: G \text { BornCoarse } \stackrel{\mathcal{X} \operatorname{Mix}_{k}^{G}}{\longrightarrow} \mathbf{M i x}_{\infty} \stackrel{\operatorname{Tot}(\mathcal{B}-)}{\longrightarrow} \mathbf{C h}_{\infty}
$$

involving composition with the functor $\operatorname{Tot}(\mathcal{B}-)(2.5)$ is $G$-equivariant coarse cyclic homology.

The definitions are justified by the following:

Theorem 3.11 The functors

$\mathcal{X} \mathrm{HH}_{k}^{G}: G$ BornCoarse $\rightarrow \mathbf{C h}_{\infty} \quad$ and $\quad \mathcal{X} \mathrm{HC}_{k}^{G}: G$ BornCoarse $\rightarrow \mathbf{C h}_{\infty}$ are G-equivariant $\mathbf{C h}_{\infty}$-valued coarse homology theories. 
Proof By Theorem 3.2, the functor $\mathcal{X} \mathrm{Mix}_{k}^{G}: G$ BornCoarse $\rightarrow \mathbf{M i x} \mathbf{x}_{\infty}$ is an equivariant coarse homology theory. The functors forget: $\mathbf{M i x} \mathbf{x}_{\infty} \rightarrow \mathbf{C h}_{\infty}$ and the functor $\operatorname{Tot}(\mathcal{B}-): \mathbf{M i x}_{\infty} \rightarrow \mathbf{C h}_{\infty}$ commute with filtered colimits and send cofiber sequences to cofiber sequences. Hence the two compositions with $\mathcal{X} \operatorname{Mix}_{k}^{G}$ satisfy coarse invariance, coarse excision, $u$-continuity, and vanishing on flasques.

The category of mixed complexes has a natural symmetric monoidal structure induced by tensor products between the underlying chain complexes [19]. As tensor products of mixed complexes preserve equivalences, $k$ being a field, we get a symmetric monoidal $\infty$-category $\mathbf{M i x}_{\infty}^{\otimes}:=\mathrm{N}\left(\mathbf{M i x}^{\otimes}\right)\left[W_{\text {mix }}^{\otimes,-1}\right] \rightarrow \mathrm{N}\left(\mathbf{F i n}_{*}\right)$ (with monoidal structure induced by the monoidal structure on Mix by [16, Proposition 3.2.2]). By [19, Theorem 2.4], the functor Mix has a lax symmetric monoidal refinement (see also [12]). This implies that coarse Hochschild and cyclic homologies are lax symmetric monoidal functors:

Proposition 3.12 The functors $\mathcal{X} \mathrm{HH}_{k}^{G}$ and $\mathcal{X} \mathrm{HC}_{k}^{G}$ admit lax symmetric monoidal refinements:

$$
\mathcal{X} \mathrm{HH}_{k}^{G, \otimes}: \mathrm{N}\left(G \text { BornCoarse }^{\otimes}\right) \rightarrow \mathbf{C h}_{\infty}^{\otimes}
$$

and

$$
\mathcal{X} \mathrm{HC}_{k}^{G, \otimes}: \mathrm{N}\left(G \text { BornCoarse }^{\otimes}\right) \rightarrow \mathbf{C h}_{\infty}^{\otimes},
$$

where $\mathbf{C h}_{\infty}^{\otimes}$ is the $\infty$-category of chain complexes with its standard symmetric monoidal structure.

Proof By [1, Theorem 3.26] and [19, Theorem 2.4], the functor $\mathcal{X} \mathrm{Mix}_{k}^{G}$ of Definition 3.1 admits a lax symmetric monoidal refinement

$$
\mathcal{X} \operatorname{Mix}_{k}^{G, \otimes}: \mathrm{N}\left(G \text { BornCoarse }^{\otimes}\right) \rightarrow \text { Mix }_{\infty}^{\otimes} .
$$

As the functors forget in (2.4) and $\operatorname{Tot}(\mathcal{B}-)$ in (2.5) are lax symmetric monoidal, coarse Hochschild and cyclic homology admit lax symmetric monoidal refinements as well.

\subsection{Comparison results and assembly maps}

In this subsection, we compare equivariant coarse Hochschild homology with the classical version of Hochschild homology for $k$-algebras. Furthermore, we show that the forget-control map for coarse Hochschild homology is equivalent to the associated generalized assembly map.

Notation 3.13 Let $A$ be a k-algebra. We denote by

$$
C_{*}^{\mathrm{HH}}(A ; k) \quad \text { and } \quad C_{*}^{\mathrm{HC}}(A ; k)
$$


the chain complexes computing the Hochschild and cyclic homology of the mixed complex $\operatorname{Mix}(A)$ associated to the cyclic object $Z_{*}(A)$ associated to $A[15,22]$.

Let $\{*\}$ be the one-point bornological coarse space, endowed with a trivial $G$-action.

Proposition 3.14 There are equivalences of chain complexes

$$
\mathcal{X} \mathrm{HH}_{k}(*) \simeq C_{*}^{\mathrm{HH}}(k ; k) \text { and } \quad \mathcal{X} \mathrm{HC}_{k}(*) \simeq C_{*}^{\mathrm{HC}}(k ; k)
$$

between the coarse Hochschild (cyclic) homology of the point and the classical Hochschild (cyclic) homology of $k$.

Proof By Theorem 2.16, the mixed complex $\operatorname{Mix}(A)$ associated to a $k$-algebra $A$ is equivalent to the mixed complex associated to the $k$-linear category of finitely generated projective $A$-modules. When $X$ is a point endowed with a trivial $G$-action and $k$ is a field, the $k$-linear category $V_{k}(X)$ is isomorphic to the category $\operatorname{Vect}_{k}^{\text {f.d. }}$ of finite-dimensional $k$-vector spaces, i.e., $\operatorname{Mix}\left(V_{k}(\{*\})\right) \simeq \operatorname{Mix}\left(\operatorname{Vect}_{k}^{\mathrm{f} . d .}\right) \simeq \operatorname{Mix}(k)$.

Let $G$ be a group. By Example 1.2, there is a canonical $G$-bornological coarse space $G_{\text {can } \min }=\left(G, \mathcal{C}_{\text {can }}, \mathcal{B}_{\text {min }}\right)$ associated to it.

Proposition 3.15 There are equivalences of chain complexes:

$$
\mathcal{X} \mathrm{HH}_{k}^{G}\left(G_{\mathrm{can}, \min }\right) \simeq C_{*}^{\mathrm{HH}}(k[G] ; k)
$$

and

$$
\mathcal{X} \mathrm{HC}_{k}^{G}\left(G_{\mathrm{can}, \min }\right) \simeq C_{*}^{\mathrm{HC}}(k[G] ; k)
$$

between the G-equivariant coarse Hochschild and cyclic homologies of $G_{\mathrm{can}, \min }$ and the classical Hochschild and cyclic homologies of the group algebra $k[G]$.

Proof The category $V_{k}^{G}\left(G_{\text {can,min }}\right)$ of $G$-equivariant $G_{\text {can,min-controlled finite- }}$ dimensional $k$-vector spaces is equivalent to the category $\operatorname{Mod}^{\mathrm{fg} \text {, free }}(k[G])$ of finitely generated free $k[G]$-modules [4, Proposition 8.24]. By Theorem 2.16, Keller's mixed complex $\operatorname{Mix}\left(\operatorname{Mod}^{\text {fg, free }}(k[G])\right)$ of the category of finitely generated free $k[G]$-modules is equivalent to the mixed complex associated to the category $\operatorname{Mod}^{\mathrm{fg}, \operatorname{proj}}(k[G])$ of finitely generated projective modules (because they are Morita equivalent dg-categories). Therefore, the result follows from the chain of equivalences of mixed complexes

$$
\operatorname{Mix}\left(V_{k}^{G}(G)\right) \simeq \operatorname{Mix}\left(\operatorname{Mod}^{\mathrm{fg}, \text { free }}(k[G])\right) \simeq \operatorname{Mix}\left(\operatorname{Mod}^{\mathrm{fg}, \operatorname{proj}}(k[G])\right) \simeq \operatorname{Mix}(k[G]),
$$

where the last equivalence is again true by Theorem 2.16 .

Let $X$ be a $G$-set and let $X_{\min , \max }$ denote the $G$-bornological coarse space with minimal coarse structure and maximal bornology. 
Remark 3.16 Let $H$ be a subgroup of $G$; then, by [4, Proposition 8.24] we get an equivalence of chain complexes:

$$
\mathcal{X} \mathrm{HH}_{k}^{G}\left((G / H)_{\min , \max } \otimes G_{\mathrm{can}, \min }\right) \simeq C_{*}^{\mathrm{HH}}(k[H] ; k) ;
$$

the same holds for equivariant coarse cyclic homology.

One of the main applications of coarse homotopy theory is the study of assembly maps. We conclude this subsection with a comparison result between the forget-control maps for equivariant coarse Hochschild and cyclic homology and the associated assembly maps. Recall the definitions of the cone functor $\mathcal{O}_{\text {hlg }}^{\infty}$ [4, Definition 10.10], of the forget-control map $\beta$ [4, Definition 11.10] and of the coarse assembly map $\alpha$ [4, Definition 10.24]. By [4, Theorem 11.16], the forget-control map for a $G$-equivariant coarse homology theory $E$ can be compared with the classical assembly map for the associated $G$-equivariant homology theory $E \circ \mathcal{O}_{h l g}^{\infty}: G \mathbf{T o p} \rightarrow \mathbf{C}$.

By applying the Eilenberg-MacLane correspondence (1.3), we can assume that the equivariant coarse homology theories $\mathcal{X} \mathrm{HH}_{k}^{G}$ and $\mathcal{X} \mathrm{HC}_{k}^{G}$ are equivariant spectravalued coarse homology theories.

Definition 3.17 Let $\mathbf{H H} \mathbf{H}_{k}^{G}:=\mathcal{E} \mathcal{M} \circ \mathcal{X} \mathrm{HH}_{k}^{G} \circ \mathcal{O}_{\mathrm{hlg}}^{\infty}: G \mathbf{T o p} \rightarrow \mathbf{S p}$ be the $G$-equivariant homology theory associated to equivariant coarse Hochschild homology.

Let Fin be the family of finite subgroups of $G$. The following is a consequence of [4, Theorem 11.16] (see also [8, Proposition 4.2.7]):

Proposition 3.18 The forget-control map $\beta_{G_{\mathrm{can}, \min }, G_{\max , \max }}$ for $\mathcal{X} \mathrm{HH}_{k}^{G}$ is equivalent to

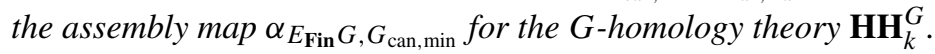

Furthermore, the assembly map $\alpha_{E_{\mathrm{Fin}} G, G_{\mathrm{can}, \min }}$ for the $G$-homology theory $\mathbf{H H}_{k}^{G}$ (hence, the forget-control map $\beta_{G_{\text {can, min }}, G_{\text {max } \max }}$ for $\mathcal{X} \mathrm{HH}_{k}^{G}$ ) is split injective by [23, Theorem 1.7].

\section{From coarse algebraic $K$-theory to coarse ordinary homology}

In this section we define a natural transformation

$$
\Phi_{\mathcal{X} \mathrm{HH}_{k}^{G}}: \mathcal{X} \mathrm{HH}_{k}^{G} \longrightarrow \mathcal{X} \mathrm{H}^{G}
$$

from equivariant coarse Hochschild homology $\mathcal{X} \mathrm{HH}_{k}^{G}$ to the $\mathbf{C h}_{\infty}$-valued equivariant coarse ordinary homology $\mathcal{X} \mathrm{H}^{G}$ and, analogously, a natural transformation $\Phi_{\mathcal{X} \mathrm{HC}_{k}^{G}}$ from equivariant coarse cyclic homology. By abuse of notation we will denote by $\mathcal{X} \mathrm{HH}^{G}, \mathcal{X} \mathrm{HC}^{G}$ and $\mathcal{X} \mathrm{H}^{G}$ both the chain and spectra valued coarse homology theories.

The transformation $\Phi_{\mathcal{X} \mathrm{HH}_{k}^{G}}$ is constructed in the following steps:

- For every $G$-bornological coarse space $X$, we consider its associated $k$-linear category $V_{k}^{G}(X)$ of controlled objects, hence the associated additive cyclic nerve 
$\mathrm{CN}\left(V_{k}^{G}(X)\right)$. For every tensor element $A_{0} \otimes \ldots \otimes A_{n}$ in the additive cyclic nerve of $V_{k}^{G}(X)$ and every $n+1$ points $x_{0}, \ldots, x_{n}$ of $X$, we define a trace-like map, which gives an element of $k$ (see Notation 4.2);

- by letting $x_{0}, \ldots, x_{n}$ vary, this yields a $G$-equivariant locally finite controlled chain on $X$, i.e., an element of $\mathcal{X} C_{n}^{G}(X)$ (see Definition 4.3 and Lemma 4.4); by letting $A_{0} \otimes \ldots \otimes A_{n}$ vary we get a map $\varphi: \mathrm{CN}_{*}\left(V_{k}^{G}(X)\right) \rightarrow \mathcal{X} C_{*}^{G}(X)$ that is a chain map with respect to the differential $d=\sum(-1)^{i} d_{i}$ of $\mathrm{CN}\left(V_{k}^{G}(X)\right)$ (see Proposition 4.6);

- the additive cyclic nerve $\mathrm{CN}\left(V_{k}^{G}(X)\right)$ yields a mixed complex with the differentials $b$ and $B$ as in Remark 2.14; the chain map $\varphi$ extends to a map of mixed complexes $\tilde{\varphi}$ (see Lemma 4.7) and yields a natural transformation of equivariant coarse homology theories $\Phi_{\mathcal{X} \mathrm{HH}_{k}^{G}}: \mathcal{X} \mathrm{HH}_{k}^{G} \longrightarrow \mathcal{X} \mathrm{H}^{G}$ (see Theorem 4.8).

We now proceed with the precise construction.

Let $V_{k}^{G}(X)$ be the $k$-linear category of $X$-controlled finite-dimensional $k$-vector spaces of Definition 1.10. The additive cyclic nerve associated to $V_{k}^{G}(X)$ (see Definition 2.13) is described, in degree $n$, by

$$
\mathrm{CN}_{n}\left(V_{k}^{G}(X)\right)=\underset{\left(\left(M_{0}, \rho_{0}\right), \ldots,\left(M_{n}, \rho_{n}\right)\right)}{ }\left(\bigotimes_{i=0}^{n} \operatorname{Hom}\left(\left(M_{i+1}, \rho_{i+1}\right),\left(M_{i}, \rho_{i}\right)\right)\right)
$$

where the index $i$ runs cyclically in the set $\{0, \ldots, n\}$ and the sum ranges over all the $(n+1)$-tuples $\left(\left(M_{0}, \rho_{0}\right), \ldots,\left(M_{n}, \rho_{n}\right)\right)$ of objects of $V_{k}^{G}(X)$.

Remark 4.1 For every controlled morphism $A_{i}:\left(M_{i+1}, \rho_{i+1}\right) \rightarrow\left(M_{i}, \rho_{i}\right)$ (see Definition 1.12) in $\operatorname{Hom}\left(\left(M_{i+1}, \rho_{i+1}\right),\left(M_{i}, \rho_{i}\right)\right)$ and for every pair of points $x$ and $y$ of $X$, there is a well-defined $k$-linear map

$$
A_{i}^{x, y}: M_{i+1}(x) \rightarrow M_{i}(y)
$$

induced by $A_{i}$.

We use the following notation:

Notation 4.2 Let $A_{0} \otimes \cdots \otimes A_{n}$ be an element of $\bigotimes_{i=0}^{n} \operatorname{Hom}\left(\left(M_{i+1}, \rho_{i+1}\right),\left(M_{i}, \rho_{i}\right)\right)$ and let $\left(\left(M_{0}, \rho_{0}\right), \ldots,\left(M_{n}, \rho_{n}\right)\right)$ be an $(n+1)$-tuple of objects of $V_{k}^{G}(X)$. Let $\left(x_{0}, \ldots, x_{n}\right)$ be a point of $X^{n+1}$. The symbol

$$
\left(A_{0} \circ \cdots \circ A_{n}\right) \mid\left(x_{0}, \ldots, x_{n}\right)
$$

denotes the linear operator $\left(A_{0} \circ \cdots \circ A_{n}\right) \mid\left(x_{0}, \ldots, x_{n}\right): M_{0}\left(x_{n}\right) \rightarrow M_{0}\left(x_{n}\right)$ defined as the composition

$$
\begin{aligned}
& \left(A_{0} \circ \cdots \circ A_{n}\right) \mid\left(x_{0}, \ldots, x_{n}\right):= \\
& \quad M_{0}\left(x_{n}\right) \stackrel{A_{n}^{x_{n}, x_{n-1}}}{\longrightarrow} M_{n}\left(x_{n-1}\right) \stackrel{A_{n-1}^{x_{n-1}, x_{n-2}}}{\longrightarrow} \ldots \stackrel{A_{1}^{x_{1}, x_{0}}}{\longrightarrow} M_{1}\left(x_{0}\right) \stackrel{A_{0}^{x_{0}, x_{n}}}{\longrightarrow} M_{0}\left(x_{n}\right)
\end{aligned}
$$


of the induced operators $A_{i}^{x_{i}, x_{i+1}}: M_{i}\left(x_{i}\right) \rightarrow M_{i+1}\left(x_{i+1}\right)$. It is an endomorphism of $M_{0}\left(x_{n}\right)$, which is a finite-dimensional $k$-vector space.

Let $X$ be a $G$-bornological coarse space and let $\mathcal{X} C_{n}(X)$ be the $k$-vector space generated by the locally finite controlled $n$-chains on $X$ (see Definition 1.6).

Definition 4.3 We let $\varphi_{n}: \mathrm{CN}_{n}\left(V_{k}^{G}(X)\right) \rightarrow \mathcal{X} C_{n}(X)$ be the map defined on elementary tensors as

$$
\begin{aligned}
& \varphi_{n}: A_{0} \otimes \cdots \otimes A_{n} \longmapsto \\
& \sum_{\left(x_{0}, \ldots, x_{n}\right) \in X^{n+1}} \operatorname{tr}\left(\left(A_{0} \circ \cdots \circ A_{n}\right) \mid\left(x_{0}, \ldots, x_{n}\right): M_{0}\left(x_{n}\right) \rightarrow M_{0}\left(x_{n}\right)\right) \cdot\left(x_{0}, \ldots, x_{n}\right)
\end{aligned}
$$

and extended linearly.

Lemma 4.4 The n-chain $\varphi_{n}\left(A_{0} \otimes \cdots \otimes A_{n}\right)$ is locally finite and controlled.

Proof In order to prove that $\varphi_{n}\left(A_{0} \otimes \cdots \otimes A_{n}\right)$ is locally finite and controlled we show that its support $\operatorname{supp}\left(\varphi_{n}\left(A_{0} \otimes \cdots \otimes A_{n}\right)\right)$ defined in (1.2) is locally finite and that there exists an entourage $U$ of $X$ such that every $x=\left(x_{0}, \ldots, x_{n}\right)$ in $\operatorname{supp}\left(\varphi_{n}\left(A_{0} \otimes \cdots \otimes A_{n}\right)\right)$ is $U$-controlled.

We first observe that the operators $A_{i}:\left(M_{i+1}, \rho_{i+1}\right) \rightarrow\left(M_{i}, \rho_{i}\right)$ are $U_{i}$-controlled for some entourage $U_{i}$ of $X$. By Definition 1.12, $A_{i}$ is given by a natural transformation of functors $M_{i+1} \rightarrow M_{i} \circ U_{i}$ [-] satisfying an equivariance condition. For every point $x$ in $X, A_{i}$ restricts to a morphism

$$
M_{i+1}(x) \rightarrow M_{i}\left(U_{i}[x]\right) \cong \bigoplus_{x^{\prime} \in U_{i}[x]} M_{i}\left(x^{\prime}\right)
$$

where the direct sum has only finitely many non-zero summands.

Let $K$ be a bounded set of $X$. The set of points $x_{n} \in K$ for which $M_{0}\left(x_{n}\right)$ is non-zero is finite (as a consequence of Definition 1.13). For such a fixed $x_{n}$, there are only finitely many points $x_{n-1} \in U_{n}[K]$ such that the corresponding map $A_{n}^{x_{n}, x_{n-1}}: M_{0}\left(x_{n}\right) \rightarrow M_{n}\left(x_{n-1}\right)$ is non-zero. The set $U_{n}[K]$ is a bounded set of $X$, the morphism $A_{n-1}: M_{n} \rightarrow M_{n-1}$ is $U_{n-1}$-controlled and we can repeat the same argument for $A_{n-1}$, hence for each $A_{i}$. This implies that the $n$-chain is locally finite because, for the given bounded set $K$, we have found only finitely many tuples $\left(x_{0}, \ldots, x_{n}\right)$ in the support of $\varphi_{n}\left(A_{0} \otimes \cdots \otimes A_{n}\right)$ that meet $K$.

The chain is also $U$-controlled, where $U$ is the entourage $U:=U_{0} \circ \cdots \circ U_{n}$ of $X$.

Remark 4.5 Let $X$ be a $G$-bornological coarse space. Let $(M, \rho)$ be a $G$-equivariant $X$-controlled finite-dimensional $k$-vector space and let $g$ be an element of the group $G$. Then, $\rho(g)$ (Definition 1.10) is a natural isomorphism between the functors $M$ and $g M$. The diagram 


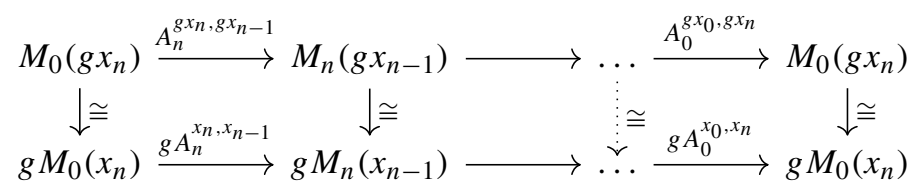

is commutative, for $A_{0} \otimes \cdots \otimes A_{n}$ in $\mathrm{CN}_{n}\left(V_{k}^{G}(X)\right)$ with $A_{i}:\left(M_{i+1}, \rho_{i+1}\right) \rightarrow$ $\left(M_{i}, \rho_{i}\right)$, where the isomorphisms are induced by $\rho_{i}(g)$. Hence, the image of $\varphi_{n}$ is a $G$-invariant locally finite controlled $n$-chain on $X$.

Let $\partial_{i}: \mathcal{X} C_{n}^{G}(X) \rightarrow \mathcal{X} C_{n-1}^{G}(X)$ be the $i$-th differential of the chain complex $\mathcal{X} C^{G}(X)$ and let $d_{i}: \mathrm{CN}_{n}\left(V_{k}^{G}(X)\right) \rightarrow \mathrm{CN}_{n-1}\left(V_{k}^{G}(X)\right)$ be the $i$-th face map of $\mathrm{CN}\left(V_{k}^{G}(X)\right)$. In the next proposition we consider the chain complex $\left(\mathrm{CN}\left(V_{k}^{G}(X)\right), d\right)$ underlying the additive cyclic nerve $\mathrm{CN}\left(V_{k}^{G}(X)\right)$.

Proposition 4.6 [8, Proposition 4.3.6] The maps $\varphi_{n}: \mathrm{CN}_{n}\left(V_{k}^{G}(X)\right) \rightarrow \mathcal{X} C_{n}^{G}(X)$ of Definition 4.3 extend to a chain map $\varphi:\left(\mathrm{CN}\left(V_{k}^{G}(X)\right), d\right) \rightarrow\left(\mathcal{X} C^{G}(X), \partial\right)$.

Proof The result is a consequence of the additivity of the trace map and of its invariance under cyclic permutations.

If $M$ is a cyclic module, as in Remark 2.14, we get a mixed complex. The chain complex $\mathcal{X} C^{G}(X)$ is also a mixed complex with the differential $B=0$.

Lemma 4.7 The chain map $\varphi: \mathrm{CN}\left(V_{k}^{G}(X)\right) \rightarrow \mathcal{X} C^{G}(X)$ of Definition 4.3 extends to a map $\tilde{\varphi}: \operatorname{Mix}\left(V_{k}^{G}(X)\right) \rightarrow \mathcal{X} C^{G}(X)$ that is a morphism of mixed complexes.

Proof The proof is a simple computation and uses the definition of the operator $B$ (2.7) and that the trace is invariant under cyclic permutations. See also [8, Lemma 4.3.7].

We can now construct the natural transformation $\Phi_{\mathcal{X} \mathrm{HH}_{k}^{G}}: \mathcal{X} \mathrm{HH}_{k}^{G} \rightarrow \mathcal{X} C^{G}$ :

Theorem 4.8 The map $\varphi$ extends to natural transformations

$$
\Phi_{\mathcal{X} \mathrm{HH}_{k}^{G}}: \mathcal{X} \mathrm{HH}_{k}^{G} \rightarrow \mathcal{X} \mathrm{H}^{G}
$$

and

$$
\Phi_{\mathcal{X} C_{k}^{G}}: \mathcal{X} \mathrm{HC}_{k}^{G} \rightarrow \bigoplus_{n \in \mathbb{N}} \mathcal{X} \mathrm{H}^{G}
$$

of G-equivariant $\mathbf{C h}_{\infty}$-valued coarse homology theories.

Proof The map $\varphi:\left(\mathrm{CN}\left(V_{k}^{G}(X)\right), d\right) \rightarrow\left(\mathcal{X} C^{G}(X), \partial\right)$ of Definition 4.3 is a chain map by Proposition 4.6. Let $f: X \rightarrow Y$ be a morphism of $G$-equivariant bornological coarse spaces. Consider the induced chain map $\mathcal{X} C^{G}(f): \mathcal{X} C^{G}(X) \rightarrow \mathcal{X} C^{G}(Y)$ and the induced functor $f_{*}=V_{k}^{G}(f): V_{k}^{G}(X) \rightarrow V_{k}^{G}\left(X^{\prime}\right)$. By functoriality of the additive 
cyclic nerve, $f_{*}$ induces a morphism $\mathrm{CN}\left(f_{*}\right): \mathrm{CN}_{*}\left(V_{k}^{G}(X)\right) \rightarrow \mathrm{CN}_{*}\left(V_{k}^{G}(Y)\right)$ of cyclic modules (hence, a chain map between the underlying chain complexes as well).

The diagram

$$
\begin{array}{rrr}
\mathrm{CN}_{n}\left(V_{k}^{G}(X)\right) & \stackrel{\varphi_{n}}{\longrightarrow} \mathcal{X} C_{n}^{G}(X) \\
\downarrow^{\mathrm{CN}\left(f_{*}\right)} & & \downarrow \mathcal{X} C^{G}(f) \\
\mathrm{CN}_{n}\left(V_{k}^{G}(Y)\right) & \stackrel{\varphi_{n}}{\longrightarrow} \mathcal{X} C_{n}^{G}(Y)
\end{array}
$$

is commutative. The map $\varphi$ extends to the associated mixed complexes by Lemma 4.7 and this extension preserves the commutative diagram (of associated mixed complexes). After localization and application of the forgetful functor (recall the definition of equivariant coarse Hochschild homology in terms of $\mathcal{X} \mathrm{Mix}_{k}^{G}$, Definition 3.10), the map $\varphi$ yields a natural transformation of equivariant coarse homology theories $\Phi_{\mathcal{X} \mathrm{H}_{k}^{G}}: \mathcal{X} \mathrm{HH}_{k}^{G} \rightarrow \mathcal{X} \mathrm{H}^{G}$.

To every mixed complex $C$, we associate the chain complex $\operatorname{Tot}(\mathcal{B} C)(2.3)$ defined by $\operatorname{Tot}_{n}(\mathcal{B C})=\bigoplus_{i \geq 0} C_{n-2 i}$ with differential $d\left(c_{n}, c_{n-2}, \ldots\right)=\left(b c_{n}+B c_{n-2}, \ldots\right)$. By Lemma 4.7, we conclude that the map $\varphi$ extends to a chain map on the total complex as well, and to a natural transformation of coarse homology theories

$$
\Phi_{\mathcal{X} \mathrm{HC}_{k}^{G}}: \mathcal{X} \mathrm{HC}_{k}^{G} \rightarrow \bigoplus_{n \in \mathbb{N}} \mathcal{X} \mathrm{H}^{G}
$$

Here the sum is indexed by the natural numbers because the (mixed complex associated to) the additive cyclic nerve of $V_{k}^{G}(X)$ is positively graded.

The following result implies that the transformation $\Phi_{\mathcal{X} \mathrm{HH}_{k}}: \mathcal{X} \mathrm{HH}_{k} \rightarrow \mathcal{X} \mathrm{H}$ is non-trivial:

Proposition 4.9 If $X$ is the one-point space $\{*\}$, then the transformation

$$
\Phi_{\mathcal{X} H_{k}}: \mathcal{X} \mathrm{HH}_{k}(*) \rightarrow \mathcal{X H}(*)
$$

induces an equivalence of chain complexes.

Proof Let $c:\{*\}^{n+1} \rightarrow k$ be an $n$-chain in $\mathcal{X} C_{n}(*)$; we identify this chain with the element $c \in k$ that is its image. Let $\left.\iota_{n}: \mathcal{X} C_{n}(*) \rightarrow \mathrm{CN}_{n}\left(V_{k}(*)\right)\right)$ be the map sending $c$ to the element $(\cdot c) \otimes\left(\cdot 1_{k}\right) \otimes \cdots \otimes\left(\cdot 1_{k}\right)$. This extends to a chain map that gives a section of the trace map, i.e., $\varphi \circ \iota=\mathrm{id}$.

As coarse Hochschild homology and coarse ordinary homology of the point are both isomorphic to the Hochschild homology of the ground field $k$ (by Example 1.8 and Proposition 3.14), we get equivalences of chain complexes

$$
\mathcal{X} \mathrm{HH}_{k}(*) \simeq C_{*}^{\mathrm{HH}}(k) \simeq \mathcal{X} C_{k}(*)
$$

By using these equivalences and the section $\varphi \circ \iota=\mathrm{id}$, we obtain that, when $X$ is the one-point space, the transformation $\Phi_{\mathcal{X}} \mathrm{HH}_{k}$ induces an equivalence of chain complexes. 
By applying the Eilenberg-MacLane correspondence $\mathcal{E} \mathcal{M}$ (1.3), we now assume that equivariant coarse Hochschild and cyclic homology take values in the $\infty$-category Sp of spectra. The classical trace map constructed by McCarthy [26, Sect. 4.4] extends to a transformation from equivariant coarse algebraic $K$-homology to equivariant coarse Hochschild homology:

\section{Proposition 4.10 [8, Proposition 4.4.1] There are natural transformations}

$$
\mathrm{K} \mathcal{X}_{k}^{G} \rightarrow \mathcal{X H H}_{k}^{G} \quad \text { and } \quad \mathrm{KX}_{k}^{G} \rightarrow \mathcal{X H C}_{k}^{G}
$$

induced by the Dennis trace maps from algebraic K-theory to Hochschild homology.

In particular, when $X$ is the $G$-bornological coarse space $G_{\text {can,min }}$, the induced map

$$
K \mathcal{X}_{k}^{G}\left(G_{\mathrm{can}, \min }\right) \rightarrow \mathcal{X} \mathrm{HH}_{k}^{G}\left(G_{\mathrm{can}, \min }\right)
$$

is the classical Dennis trace map $K(k[G]) \rightarrow \mathrm{HH}(k[G])$ by McCarthy's agreement result [26, Sect. 4.5], by [4, Proposition 8.24] and by Proposition 3.15.

Composing the transformations of Proposition 4.10 and of Theorem 4.8 we get the natural transformation

$$
K \mathcal{X}_{k}^{G} \rightarrow \mathcal{X} \mathrm{HH}_{k}^{G} \stackrel{\Phi_{\mathcal{X H H}}^{G}}{\longrightarrow} \mathcal{X} \mathrm{H}_{k}^{G}
$$

from equivariant coarse algebraic $K$-homology to equivariant coarse ordinary homology. When $X$ is the $G$-bornological coarse space $G_{\text {can,min }}$, we get a map $K(k[G]) \rightarrow$ $H(G ; k)$ from the algebraic $K$-theory of the group ring $k[G]$ to the ordinary homology of $G$ with $k$-coefficients. We believe that further investigations of this transformation can be useful to detect coarse $K$-theory classes.

Acknowledgements Open Access funding provided by Projekt DEAL. This work formed part of the author's PhD thesis at Regensburg University. It is a pleasure to again acknowledge Ulrich Bunke, this work would not exist without him. The author also thanks Clara Löh, Denis-Charles Cisinski and Alexander Engel for helpful discussions, and the anonymous referees for constructive comments and recommendations. The author has been supported by the DFG Research Training Group GRK 1692 "Curvature, Cycles, and Cohomology" and by the DFG SFB 1085 "Higher Invariants".

Open Access This article is licensed under a Creative Commons Attribution 4.0 International License, which permits use, sharing, adaptation, distribution and reproduction in any medium or format, as long as you give appropriate credit to the original author(s) and the source, provide a link to the Creative Commons licence, and indicate if changes were made. The images or other third party material in this article are included in the article's Creative Commons licence, unless indicated otherwise in a credit line to the material. If material is not included in the article's Creative Commons licence and your intended use is not permitted by statutory regulation or exceeds the permitted use, you will need to obtain permission directly from the copyright holder. To view a copy of this licence, visit http://creativecommons.org/licenses/by/4.0/.

\section{References}

1. Bunke, U., Caputi, L.: Controlled objects as a symmetric monoidal functor, arXiv e-prints, arXiv:1902.03053 (2019) 
2. Bunke, U., Cisinski, D.-C.: A universal coarse $K$-theory. N. Y. J. Math. 26, 1-27 (2020)

3. Bunke, U., Engel, A., Kasprowski, D., Winges, C.: Equivariant coarse homotopy theory and coarse algebraic K-homology. In: Guillermo, C., Weibel, C.A. (eds.) K-theory in algebra, analysis and topology, pp. 13-104. American Mathematical Society, Providence, RI (2020). https://doi.org/10.1090/ conm/749

4. Bunke, U., Engel, A., Kasprowski, D., Winges, C.: Equivariant coarse homotopy theory and coarse algebraic $K$-homology, K-theory in algebra, analysis and topology, Contemp. Math. 749, 13-104 (2020)

5. Bökstedt, M., Hsiang, W.C., Madsen, I.: The cyclotomic trace and algebraic $K$-theory of spaces. Invent. Math. 111(3), 465-539 (1993). 1202133

6. Bunke, U., Kasprowski, D., Winges, C.: Split injectivity of a-theoretic assembly maps. In: International Mathematics Research Notices, (2019) http://oup.prod.sis.lan/imrn/advance-article-pdf/doi/10.1093/ imrn/rnz209/30252206/rnz209.pdf. Accessed 2019

7. Bartels, A., Reich, H.: Coefficients for the farrell-jones conjecture. Adv. Math. 209(1), 337-362 (2007)

8. Caputi, L.: Hochschild and cyclic homology for bornological coarse spaces, (2019) https://epub.uniregensburg.de/40219/. Accessed 2019

9. Cisinski, D.-C.: Higher categories and homotopical algebra,http://www.mathematik.uni-regensburg. de/cisinski/CatLR.pdf

10. Cohn, L.: Differential graded categories are k-linear stable infinity categories, (2013) arXiv:1308.2587

11. Cárdenas, M., Pedersen, E.K.: On the Karoubi filtration of a category. K-theory 12, 165-191 (1997)

12. Cisinski, D.-C., Tabuada, G.: Symmetric monoidal structure on non-commutative motives. J. K-Theory 9(2), 201-268 (2012). 2922389

13. Dundas, B.I., Goodwillie, T., McCarthy, R.: The local structure of algebraic $K$-theory, vol. 18. Springer, Berlin (2013)

14. Faonte, G.: Simplicial nerve of an $A_{\infty}$-category. Theory Appl. Categ. 32(31-52), 3607208 (2017)

15. Goodwillie, T.G.: Cyclic homology, derivations, and the free loopspace. Topology 24(2), 187-215 (1985)

16. Hinich, V.: Dwyer-Kan localization revisited. Homol. Homot. Appl. 18(1), 27-48 (2016). 3460765

17. Karoubi, M.: Foncteurs derivés et $K$-theorie. Springer, Berlin Heidelberg (1970)

18. Kasprowski, D.: On the $K$-theory of groups with finite decomposition complexity. Proc. Lond. Math. Soc. 110(3), 565-592 (2015)

19. Kassel, C.: Cyclic homology, comodules, and mixed complexes. J. Algebra 107(1), 195-216 (1987)

20. Keller, B.: On differential graded categories. Int. Cong. Math. 2, 151-190 (2006)

21. Keller, B.: On the cyclic homology of exact categories. J. Pure Appl. Algebra 136(1), 1-56 (1999)

22. Loday, J.-L.: Cyclic homology, Grundlehren der mathematischen Wissenschaften, vol. 301. Springer, Berlin Heidelberg (1998)

23. Lück, W., Reich, H.: Detecting $K$-theory by cyclic homology. Proc. Lond. Math. Soc. (3) 93(3), 593-634 (2006). 2266961

24. Lurie, J.: Higher topos theory, annals of mathematics studies, vol. 170. Princeton University Press, Princeton (2009)

25. Lurie, J., Higher algebra, (2014), Available at www.math.harvard.edu/lurie. Accessed 2014

26. McCarthy, R.: The cyclic homology of an exact category. J. Pure Appl. Algebra 93(3), 251-296 (1994)

27. Mitchener, P.D.: Coarse homology theories. Algebr. Geom. Topol. 1, 271-297 (2001)

28. Roe, J.: Lectures on coarse geometry, University Lecture Series, vol. 31. pp. viii+175. American Mathematical Society, Providence, RI (2003)

29. Roe, J.: Coarse cohomology and index theory on complete riemannian manifolds. Mem. Am. Math. Soc. 104, 497 (1993)

30. Roe, J.: Index theory, coarse geometry, and topology of manifolds. In: CBMS Regional Conference Series in Mathematics, vol. 90. pp. $x+100$. American Mathematical Society, Providence, RI (1996)

31. Schlichting, M.: Delooping the $K$-theory of exact categories. Topology 43(5), 1089-1103 (2004)

32. Schlichting, M.: Higher Algebraic $K$-Theory (After Quillen Thomason and Others). Springer, Berlin Heidelberg (2011)

33. Shipley, B.: $H \mathbb{Z}$-algebra spectra are differential graded algebras. Am. J. Math. 129(2), 351-379(2007). MR 2306038

34. Tabuada, G.: Invariants additifs de dg-catégories. Internat. Math. Res. Not 53, 3309-3339 (2005)

35. Tabuada, G.: Theorie homotopique des DG-categories, Ph.D. thesis (2007). arXiv:0710.4303 
Publisher's Note Springer Nature remains neutral with regard to jurisdictional claims in published maps and institutional affiliations. 